\title{
Tunnels profonds soumis à un chargement thermique
}

\section{H. WONG \\ G. ROUSSET}

Groupement pour l'étude des structures souterraines de stockage (G.3S/LMS)

École Polytechnique 91128 Palaiseau Cedex
En condition isotherme, la méthode classique Convergence-Confinement fournit un moyen efficace d'étude de prédimensionnement des tunnels profonds. Pour le cas particulier du stockage de substances exothermiques, les élévations de température engendrent des contraintes mécaniques qui peuvent devenir significatives. Cette composante thermique doit donc être prise en compte dans le dimensionnement des ouvrages souterrains. Dans cet article, on présente un modèle analytique du comportement d'un tunnel profond dans un massif élasto-plastique, en conditions non isothermes. Moyennant des hypothèses simplificatrices mais réalistes, on aboutit à une solution dont les expressions sont explicites; seule la détermination des frontières séparant les différentes zones élasto-plastiques nécessite une petite manipulation numérique, pour résoudre un système d'équations algébriques. Grâce à sa simplicité, cette solution analytique permet de mettre en évidence d'une manière pertinente l'influence du chargement thermique, et de dégager des conclusions utiles pour le dimensionnement des tunnels profonds soumis à chargement thermique.

Par ailleurs, la méthode Convergence-Confinement étant largement utilisée dans le domaine de la géotechnique, il paraît intéressant d'interpréter les résultats issus du modèle analytique sous l'angle de cette méthode. Le dernier paragraphe présente les premiers résultats de cet effort de rapprochement.

\section{Deep tunnels under thermal loading}

Under isothermal conditions, the Convergence Confinement method furnishes an efficient means for the preliminary design and dimensioning of deep tunnels. For the particular case of the underground storage of exothermic substances, the temperature rises provoke mechanical stresses that may become significant. This thermal component must therefore be taken account of in the dimensioning of the underground storage structures. In this paper, we present an analytical model on the behaviour of deep tunnels in an elastoplastic medium, under non-isothermal conditions. Based on a set of simplifying but realistic assumptions, we arrive at a solution with totally explicite expressions. Only the determination of the plastic fronts separating different elastoplastic zones requires a little numerical manipulation to solve the set of simple algebraic equations. Thanks to its simplicity, this analytical solution allows us to put into evidence in a pertinent manner the influence of thermal loads, and to draw useful conclusions for the dimensioning of deep tunnels subject to thermal loading. Furthermore, the Convergence Confinement method being largely known and frequently applied in geotechnical engineering, it appears interesting to interpret the results obtained from the analytical model in the light of this method. The last paragraph presents the first results in this direction. 


\section{Introduction}

Le comportement d'un tunnel dans un massif infini et en condition isotherme est maintenant bien connu, notamment sous la lumière de la méthode Convergence-Confinement (Panet, 1976), (Egger, 1973), (Corbetta, Bernaud, Minh, 1991) et (Bernaud et Rousset, 1992, 1993). En raison de sa simplicité, cette méthode a connu un grand succès en géotechnique, et elle est devenue un outil indispensable pour les études préliminaires des tunnels profonds ((AFTES, 1979) puis (AFTES, 1983)).

En condition isotherme, des comportements assez complexes peuvent être envisagés (voir par exemple (Rousset, 1988) et (Minh, 1984)).

En revanche, pour certains problèmes où les phénomènes thermiques interviennent, par exemple les liaisons de gazéification souterraine du charbon (Mihn et Schmitt, 1986, 1987), les forages d'exploitation en géothermie (Goguel, 1975), ou le stockage souterrain des déchets radioactifs (Rousset, 1992), les modèles analytiques simples et efficaces sont beaucoup moins nombreux.

Parmi les premiers modèles analytiques en thermomécanique, on peut citer ceux de (Bland, 1956) et de (Mendelsen, 1968), qui analysent un tube épais élastoplastique soumis à un champ thermique stationnaire, et une pression interne variable. Le critère plastique est celui de Tresca. L'hypothèse du régime de face (la

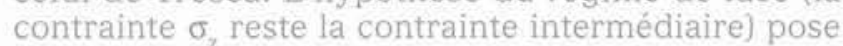
toutefois une limite supérieure sur l'élévation de température qui puisse être prise en compte.

En symétrie sphérique, on peut citer les travaux de (Bérest, 1986) sur le comportement thermoplastique d'un solide infini, sous l'effet d'une source thermique ponctuelle ou répartie dans une sphère, Ce modèle analytique est ensuite étendu au comportement thermoviscoplastique (Bérest et Rousset, 1986).

D'autres modèles, plus complexes, nécessitent des procédés numériques plus élaborés; c'est par exemple le cas du modèle thermoviscoplastique de (Ishikawa et Hata 1980), et le modèle thermoplastique de (Minh et Schmitt, 1986, 1987).

En comportement purement élastique, la résolution du problème est grandement simplifiée; des solutions analytiques existent alors également pour les milieux poreux remplis d'un fluide saturant (Booker et Savvidou, 1985) et (Giraud, 1993).

Les solutions analytiques d'une cavité soumise à un chargement thermique sont rares, surtout lorsqu'il s'agit de comportements irréversibles et dans le cas de l'axisymétrie ; ce fait peut être expliqué par deux facteurs. D'une part, les solutions thermiques analytiques en régime transitoire sont très complexes (Carslaw et Jaeger, 1960), et difficilement manipulables, le problème étant particulièrement aigu en axisymétrie. D'ailleurs, dans ce dernier cas, le régime thermique permanent n'existe pas en milieu infini en cas de chargement constant (température ou flux). D'autre part, contrairement au chargement mécanique qui agit uniquement en paroi (un scalaire), le chargement thermique (un champ), quant à lui, agit sur tout le massif : les contraintes en un point donné dépendent des températures dans l'ensemble du massif d'une manière complexe. Par conséquent, le couplage thermomécanique est intrinsèquement plus compliqué à traiter qu'un chargement purement mécanique.

Pour aboutir à une solution simple, la prise en compte des lois de comportement simples est impérative ; plasticité ou viscoplasticité associée, avec le critère de Tresca, sont les plus souvent envisagées. C'est également le cas de cet article. En contrepartie de sa simplicité de forme, la présence des coins anguleux complique l'analyse et oblige à distinguer les zones plastiques de natures différentes, suivant qu'on est en présence du régime de face ou d'arête. Enfin, on verra plus loin que la présence du chargement thermique conduit à l'apparition des zones plastiques qu'on ne rencontre pas en condition isotherme.

\section{Problème posé et hypothèses générales}

On considère une galerie circulaire profonde, de rayon a, dont la paroi est soumise à un échauffement. On se place dans le cadre d'hypothèses simples qui permet de rendre le problème unidimensionnel : massif homogène, isotrope et infini soumis initialement à un champ de contraintes $\underline{\sigma}_{\infty}=-\mathrm{P}_{\infty} \underline{=}$ homogene et isotrope ; par ailleurs, le problème peut être analysé en axisymétrie et déformation plane. Enfin, on ne considère que des petites transformations, et on suppose le problème quasi statique. A l'état initial, les déformations et les déplacements sont supposés nuls.

\section{Chargement thermomécanique}

Le creusement de la galerie constitue la première étape de chargement (chargement mécanique). Conformément à l'esprit de la méthode Convergence-Confinement ( $\mathrm{CV}$-CF), le creusement est simulé par une pression intérieure monotone décroissante à partir de

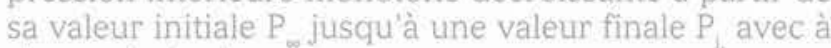
chaque instant:

$$
\dot{\mathrm{P}}_{3}(\mathrm{t})<0
$$

Ensuite, commence la seconde étape du chargement (chargement thermique), qui engendre un champ de température. Pour fixer les idées, supposons pour l'instant que la pression interne est maintenue constante pendant cette deuxième étape de chargement, c'est-à-dire $\dot{P}(t)=0$. Par ailleurs, on suppose dans la suite une distribution de température axisymétrique de la forme $T=T(r, t)$ dans un système de coordonnées cylindriques $r, \theta, z$ qui coîncident avec les directions des contraintes principales notées $\sigma_{r}, \sigma_{\theta}$ et $\sigma_{z}$; l'axe $z$ est l'axe de la galerie. Pour simplifier davantage, on suppose que ce champ thermique vérifie les deux hypothèses fondamentales suivantes (profil de température monotone décroissant et évolution monotone croissante dans le temps):

$$
\begin{aligned}
& \partial_{\mathrm{r}} \mathrm{T}<0 \\
& \partial_{\mathrm{T}} \mathrm{T}>0
\end{aligned}
$$

Notons que ces hypothèses couvrent la plupart des cas pratiques ; à titre d'exemple, les hypothèses (H2) et (H3) sont vérifiées dans les deux cas suivants : 
a) une température imposée constante $T_{0}$ en paroi ; la solution correspondante est :

$$
\begin{gathered}
\frac{T(r, t)}{T_{0}}= \\
1+\frac{2}{\pi} \int_{0}^{\infty} \frac{e^{-k u 2 t}}{u} \frac{J_{0}(u r) Y_{0}(u a)-Y_{0}(u r) J_{0}(u a)}{J_{0}^{2}(u a)+Y_{0}^{2}(u a)} d u
\end{gathered}
$$

b) un flux imposé constant $\Phi_{0}$ par unité de surface $\left(\mathrm{Wm}^{-2}\right)$ de la paroi, la solution est alors:

$$
\begin{gathered}
\frac{K}{\phi_{0}} T(r, t)= \\
-\frac{2}{\pi} \int_{0}^{\infty} \frac{1-e^{-k u^{2} t}}{u^{2}} \frac{J_{0}(u r) Y_{1}(u a)-Y_{0}(u r) J_{1}(\text { ua })}{J_{1}^{2}(u a)+Y_{1}^{2}(u a)} d u
\end{gathered}
$$

où $\mathrm{K}$ et $\mathrm{k}$ sont respectivement la conductivité et la diffusivité thermique du massif. La définition des fonctions de Bessel $J_{\gamma} J_{1}, Y_{0 y}$ et $Y_{1}$, ainsi que leurs propriétés, peuvent être trouvées par exemple dans (Hildebrand, 1977).

Lorsque le flux est exponentiellement décroissant, c'est-à-dire $\Phi(\mathrm{t})=\Phi_{0} \mathrm{e}^{-\omega t}$. la solution s'obtient à partir du cas (b) ci-dessus grâce au principe de Duhamel (Hildebrand, 1977, p. 463) :

$$
\begin{gathered}
\frac{K}{\phi_{0}} T(r, t)= \\
-\frac{2}{\pi} \int_{0}^{\infty} \frac{e^{-\omega t}-e^{-k u^{2} t}}{u^{2}-\frac{\omega}{k}} \frac{J_{0}(u r) Y_{1}(u a)-Y_{0}(u r) J_{1}(u a)}{J_{1}^{2}(u a)+Y_{1}^{2}(u a)} d u
\end{gathered}
$$

la constante de décroissance $\omega$ étant liée à la demivie $t_{\omega}$ par la formule $t_{\omega}=\frac{\log 2}{\omega}$.

Dans ce dernier cas, la condition $\partial T<0$ est à chaque instant vérifiée; par contre $\partial \mathrm{T}$ n'est positif qu'avant l'instant du paroxysme $t_{r}$ car on a : $\partial \mathrm{T}(\mathrm{a}, \mathrm{t}<\mathrm{t}$ ) $>0 ; \partial_{1} T\left(a, t=t_{p}\right)=0 ; \partial_{t} T\left(a, t>t_{p}\right)<0$. Par conséquent, le modèle développé ici ne s'applique que pour $t<t$. dans le cas d'un flux exponentiellement décroissant.

Signalons en passant que l'exploitation numérique de ces solutions thermiques analytiques n'est pas aisée, notamment en raison de l'intégrale de borne supérieure infinie et des fonctions de Bessel infiniment oscillatoires. Des développements théoriques, accompagnés de techniques numériques spécifiques sont nécessaires pour aboutir à des algorithmes fiables et performants. L'analyse théorique de ce problème particulier ne relève pas ici de notre propos. Les détails s'y rapportant peuvent être trouvés dans (Jaeger, 1956), (Ritchie et Sakakura, 1956) et (Wong, 1994).

Par ailleurs, il découle des hypothèses précédentes que le champ de déplacements, ainsi que ceux des vitesses, contraintes, et déformations, admettent les formes particulièrement simples suivantes:

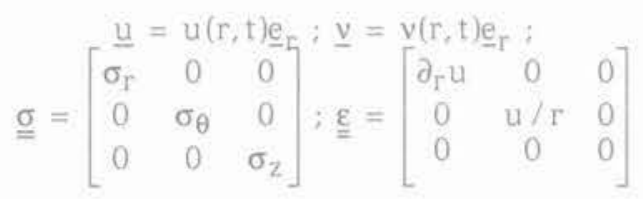

avec $v=\partial_{t} u$, et $\underline{e}_{r}=$ vecteur unitaire radial.

\section{4}

\section{Comportement du matériau}

Dans la suite, le comportement du matériau est élasto-plastique, la vitesse de déformation est la somme d'une composante élastique linéaire caractérisée par deux constantes $E$ et $v$, d'une composante thermique caractérisée par le coefficient de la dilatation thermique linéique $\alpha$, et d'une composante plastique irréversible $\varepsilon^{p}$ :

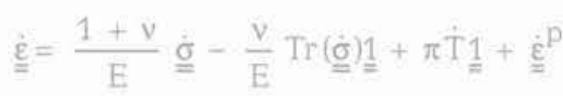

Dans cet article, on considère uniquement le critère plastique de Tresca, qui s'écrit ( $\mathrm{C}=$ cohésion du matériau) :

$$
f(\underline{\underline{\sigma}}) \equiv \sigma_{1}-\sigma_{3}-2 C=0
$$

où $\sigma_{1}>\sigma_{2}>\sigma_{3}$ sont les contraintes principales ordonnées. Il est possible que deux contraintes principales deviennent égales au cours du chargement, provo. quant ainsi l'apparition du régime d'arête. Par ailleurs, la loi d'écoulement, qui permet la détermination de $\dot{\varepsilon}^{p}$ est associée :

$$
\underline{\underline{\varepsilon}}^{p}=\dot{\lambda} \partial_{\sigma} f(\underline{\underline{\sigma}}
$$

avec $\dot{\lambda}>0$ si et seulement si $\mathrm{f}(\underline{\underline{\sigma}})=0$ et $\mathrm{f}(\underline{\underline{\sigma}})=0$, sinon $\dot{\lambda}$ $=0$. En cas de régime d'arête (par exemple $\sigma 1>\sigma 2=$ $\sigma 3)$, l'état de contrainte se trouve sur un " coin » de la surface et y reste; on doit alors sommer la contribution des deux faces adjacentes :

$$
f_{1}=\sigma_{1}-\sigma_{2}-2 C=0 ; f_{2}=\sigma_{1}-\sigma_{3}-2 C=0
$$

soit donc (voir par exemple (Halphen et Salençon, 1987) ou (Jain, 1980)) :

$$
\dot{\varepsilon}^{p}=\dot{\lambda} \partial_{\underline{\sigma}} f_{1}+\mu \partial_{\underline{\sigma}} f_{2}
$$

qui s'écrit sous forme matricielle :

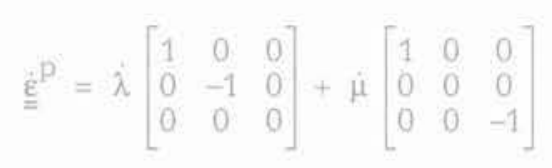

\section{5}

\section{Équations générales}

En intégrant l'équation (1) entre l'instant initial $t=0$ et un instant ultérieur quelconque $t$, on obtient alors

$$
\begin{gathered}
E^{*} \partial_{r} u= \\
\sigma_{r}^{*}-v\left(\sigma_{\theta}^{*}+\sigma_{z}^{*}\right)+2(1-v) \theta+E^{*} \lambda+E^{*} \mu+(1-2 v) P_{\infty}^{*} \\
E^{*} \frac{u}{r}= \\
\sigma_{\theta}^{*}-v\left(\sigma_{z}^{*}+\sigma_{r}^{*}\right)+2(1-v) \theta-E^{*} \lambda+E^{*} \gamma+(1-2 v) P_{\infty}^{*} \\
0= \\
\sigma_{z}^{*}-v\left(\sigma_{r}^{*}+\sigma_{\theta}^{*}\right)+2(1-v) \theta-E^{*} \mu-E^{*} \gamma+(1-2 v) P_{\infty}^{*}
\end{gathered}
$$


équations dans lesquelles $\lambda$ est la composante de $\varepsilon p$ associée au déviateur $\sigma_{r}-\sigma_{\theta}$ et $\mu$ et $\gamma$ sont associés aux déviateurs $\sigma_{r}-\sigma_{z}$ et $\sigma_{\theta}-\sigma_{z}$ respectivement ; les grandeurs physiques $\lambda$ et $\mu$ sont à distinguer des constantes de Lamé. Par ailleurs, l'équation d'équilibre s'écrit :

$$
\sigma_{\theta}^{*}-\sigma_{r}^{*}=r \partial_{r} \sigma_{r}^{*}
$$

L'écriture des équations (7) à (10) tient compte du système de notation adimensionnel suivant:

$$
\begin{gathered}
\sigma_{r}^{*} \equiv \frac{\sigma_{r}}{C} \ldots \text { etc. } ; P_{\infty}^{*} \equiv \frac{P_{\infty}}{C} ; P_{i}^{*} \equiv \frac{P_{1}}{C} \\
E^{*} \equiv \frac{E}{C} ; \theta \equiv \frac{E \alpha T}{2 C(1-v)}
\end{gathered}
$$

La définition du chargement thermique adimensionnel $\theta \equiv T / T^{*}$ fait apparaitre une température caractéristique $T^{*} \equiv 2 \mathrm{C}(1-v) /$ E $\alpha$ qui est un paramètre intrinsèque du matériau : on verra plus loin que, plus sa valeur est élevée, moins le matériau est sensible à l'effet thermique. On définit par ailleurs le chargement mécanique adimensionnel $\Delta \mathrm{P}^{*}(\mathrm{t}) \equiv \mathrm{P}^{*}{ }_{\infty}-\mathrm{P}^{*}(\mathrm{t})$, qui vérifie, en vertu de $(\mathrm{H} 1)$ :

$$
\Delta * P^{*}>0
$$

Pour alléger l'écriture, on enlèvera les étoiles par la suite. On comprendra que les grandeurs telles que E, $\sigma, \eta, P_{\infty}, P_{1}$ et $\Delta P$, sont des quantités normalisées par rapport à la cohésion.

\section{6}

\section{Évolution des zones élasto-plastiques pendant l'échauffement}

La résolution détaillée du problème peut être trouvée (Wong et Rousset, 1993). On se contente de retracer ici la succession des événements et les équations les plus significatives. La solution détaillée est résumée en annexe $\mathrm{A}$ pour une référence utile. En supposant que le chargement mécanique n'ait pas franchi le seuil de plastification du massif au moment où commence l'échauffement $(\Delta \mathrm{P}<1)$, l'évolution des diverses zones plastiques suit le scénario suivant:

\section{Phase 1-Massif entièrement élastique}

Au cours de cette première phase, le déviateur maximal $\sigma_{r}-\sigma_{\theta^{\prime}}$ qui est une fonction décroissante de $r$,
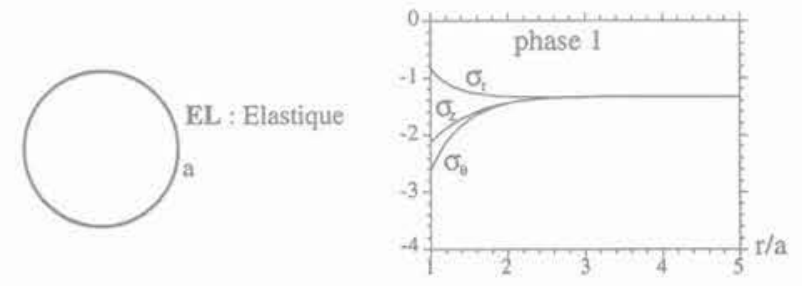

FG. 1 Allure des profils de contraintes à la phase 1 .

Shape of the stress profiles in phase 1. augmente en paroi. Cette première phase se termine donc à l'instant où le critère est atteint en paroi par le déviateur $\sigma_{\mathrm{r}}-\sigma_{\theta}$ lorsque :

$$
\Delta \mathrm{P}(\mathrm{t})+\theta(\mathrm{a}, \mathrm{t})=1
$$

C'est-à-dire lorsque la température en paroi atteint la valeur $1-\Delta \mathrm{P}(\mathrm{t})$.

\section{Phase 2 - Première zone plastique} en régime de face $\mathrm{RF} 1$
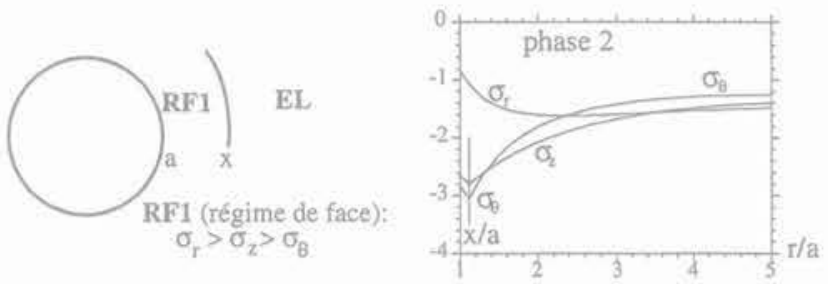

FIG \& Succession des zones élastoplastiques et profils des contraintes en phase 2. Succession of elastoplastic zones and stress profiles in phase 2.

Dans cette deuxième phase, une zone plastique en régime de face $\left(\sigma_{p}>\sigma_{7}>\sigma_{\theta}\right)$ coexiste avec la zone élastique extérieure. On notera également que l'ordre des contraintes dans la zone élastique varie plusieurs fois en fonction du rayon. L'évolution de la frontière $\mathrm{x}$ qui sépare les deux zones est décrite par l'équation algébrique suivante :

$$
\begin{gathered}
\theta(x, t)=1-\Delta P+\log \left(\frac{x}{a}\right)^{2} \equiv M(x) \\
\left.\operatorname{cou} \Omega(x, t) \equiv 1-\theta(x, t)-\Delta P(t)+\log \left(\frac{x}{a}\right)^{2}=0\right)
\end{gathered}
$$

On montre que la phase 2 se termine quand $\sigma_{\theta}$ et $\sigma_{\text {. }}$ deviennent égales en paroi. Cet événement se produit lorsque :

$$
\frac{1-2 v}{2(1-v)} \Delta P(t)+\theta(a, t)=1
$$

C'est-à-dire lorsque la température en paroi atteint la valeur $1-\frac{1-2 v}{2(1-v)} \Delta \mathrm{P}(\mathrm{t})$.

Phase 3 - Deux zones plastiques : régime de face RF1 et régime d'arête RA

Lorsque le chargement thermomécanique franchit le seuil précédent, on montre qu'une zone plastique en régime d'arête $\mathrm{RA}\left(\sigma_{r}>\sigma_{z}=\sigma_{\theta}\right)$ apparaît en paroi et se développe de façon analogue au cas isotherme traité par (Mandel, 1976).

Notons en passant qu'en condition isotherme $\theta=0$, le régime d'arête peut être évité si l'on se contente d'étudier un matériau incompressible, $(v=0,5)$, car la condition (13) ne peut alors jamais être atteinte. En revanche, en présence du chargement thermique, le régime d'arête est toujours possible, quelle que soit la valeur de $v$.

L'équation précédente sur x est toujours valable, tandis que la frontière intérieure y est déterminée par 

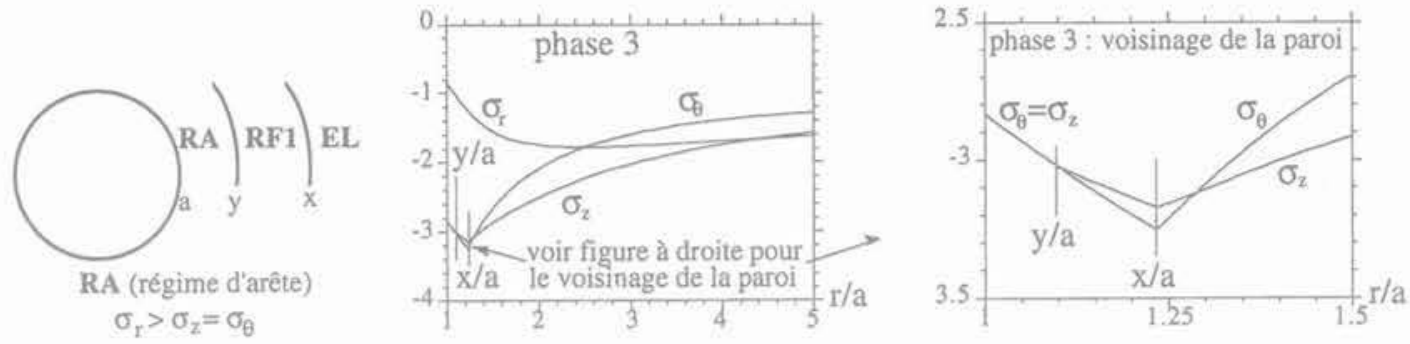

F6.3 Succession des zones élasto-plastiques et profills des contraintes en phase 3 ,

Succession of elastoplastic zones and stress profiles in phase 3.

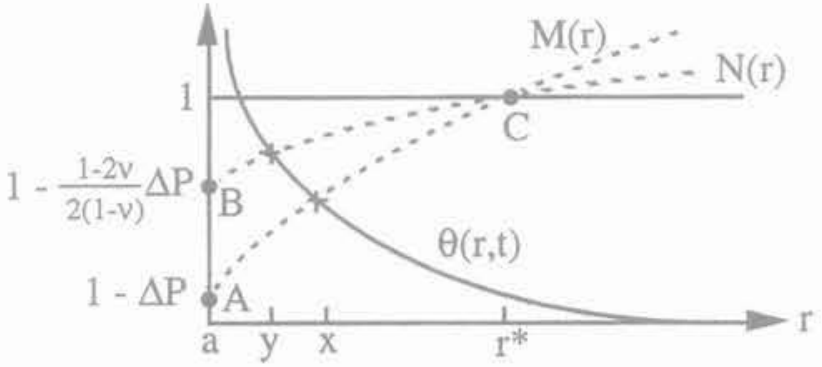

FIG. 4

Interprétation graphique des équations sur les rayons plastiques pour les trois premières phases.

Graphical interpretation of the equations on the plastics fronts of the first three phases.

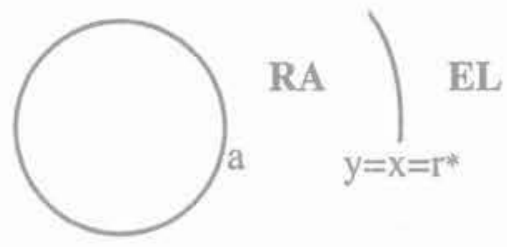

TG. 5

Succession des zones élasto-plastiques à la fin de la phase 3 .

Succession of elastoplastic zones at the end of phase 3.
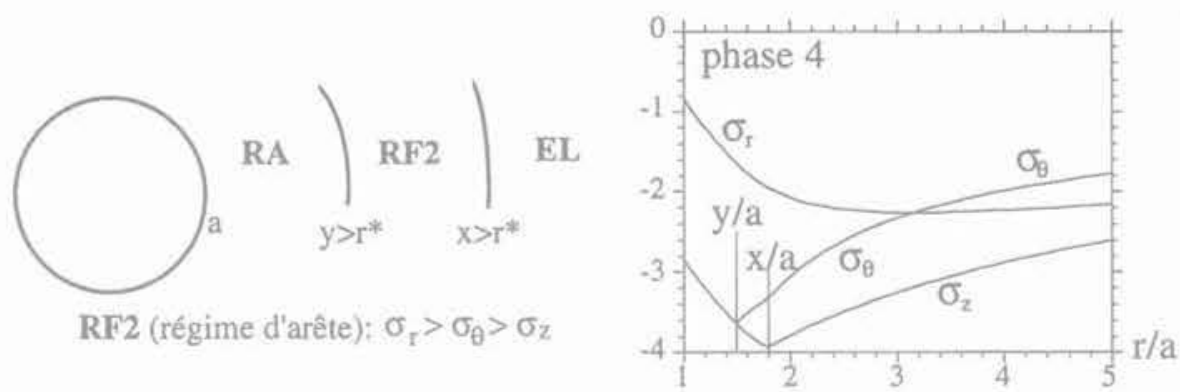

FG.6 Succession des zones élasto-plastiques et profils des contraintes en phase 4.

Succession of elastoplastic zones and stress proflies in phase 4.

une équation algébrique formellement analogue à la précédente :

$$
\theta(\mathrm{y}, \mathrm{t})=1-\frac{1-2 v}{2(1-v)}\left(\Delta \mathrm{P}-\log \left(\frac{\mathrm{y}}{\mathrm{a}}\right)^{2}\right) \equiv \mathrm{N}(\mathrm{y})(14)
$$

A titre d'interprétation graphique, les « rayons plastiques n x et y sont donnés par les intersections entre le profil de température $\theta$, qui évolue dans le temps, et deux fonctions stationnaires $\mathrm{M}$ et $\mathrm{N}$. La figure 4 illustre cette propriété.

La nature des zones plastiques dépend donc simplement de la position relative du profil q par rapport aux trois « pivots » $\mathrm{A}, \mathrm{B}$, et C:

$-\theta$ passe en deçà de $A$ : phase 1 , massif entièrement élastique ;

$-\theta$ passe entre $A$ et $B$ : phase 2 , une zone plastique RF1; $-\theta$ passe entre $B$ et $C$ : phase 3 , deux zones plastiques RF1 et RA ;
- $\theta$ passe au-delà de $\mathrm{C}$ : phase 4 , voir paragraphe suivant.

Une propriété remarquable du problème précédent est que les deux fonctions $M(r)$ et $N(r)$ se croisent au point

$C\left(r^{*}, 1\right)$ en un rayon $r^{*}=a \exp \left(\frac{\Delta P}{2}\right)$ qui dépend uni quement du chargement mécanique $\Delta \mathrm{P}$. En conséquence, quelle que soit la forme de $\theta(\mathrm{r})$, pourvu qu'elle satisfasse ( $\mathrm{H} 2)$ et $(\mathrm{H} 3)$, les deux rayons x et y doivent se joindre en $r^{*}$, lorsque à un instant $t$, la température adimensionnelle au rayon $r^{\star}$ atteint la valeur unité, c'est-àdire :

$$
\theta\left(r^{*}, t\right)=1
$$

Cet événement marque la fin de la phase 3 (la zone RF1 disparaît), comme l'illustre la figure 5. 


\section{Phase 4 - Une nouvelle zone plastique en régime de face RF2 voisine de RA}

A la fin de la phase 3 , nous sornmes dans une situation tout à fait particulière : la zone plastique RF1 est réduite à une surface cylindrique en $r=r^{*}$, et la zone en régime d'arête RA est en contact avec la zone élastique EL. Apparait maintenant une nouvelle zone plastique RF2 entre les zones RA et EL. Cette nouvelle zone plastique RF2 diffère de RF1 par l'ordre des contraintes principales, car dans RF2 doivent être vérifiées les inégalités $\sigma_{f}>\sigma_{\theta}>\sigma_{1}$. Nous pouvons remarquer que cette configuration n'apparaît qu'en présence du chargement thermique.

La résolution de cette phase est plus complexe que les autres, et les équations algébriques qui déterminent les rayons plastiques $\mathrm{x}$ et y sont cette fois-ci couplées ; leur résolution nécessite une petite manipulation numérique.

$$
\begin{gathered}
3[1-\theta(x, t)] x^{2}-[1-\theta(y, t)] y^{2}+3 \int_{y}^{x} \xi \theta(\xi, t) d \xi=0 \\
{\left[\Delta P-1+\theta(x, t)-\log \left(\frac{x}{a}\right)^{2}\right]} \\
+\left[\Delta P-1+\theta(y, t)-\log \left(\frac{y}{a}\right)^{2}\right] \\
+\int_{y}^{x} \frac{\theta(\xi, t)}{\xi} d \xi=0
\end{gathered}
$$

En toute généralité, si le chargement thermique se développe encore, de nouvelles phases doivent apparaître. La discussion sur l'achèvement de la phase 4 est difficile car plusieurs cas se présentent. Néanmoins, ces quatre phases s'avèrent suffisantes pour traiter le problème de façon satisfaisante, compte tenu de la fourchette des valeurs de températures rencontrées en pratique.

En résumé, on retiendra de la solution originale commentée ci-dessus quatre points importants

- Le chargement thermique en milieu élastoplastique modifie considérablement le champ de contrainte ; en particulier l'ordre des contraintes principales peut être modifié en chaque point:

- Ce problème complexe admet (au moins pour les premières phases) une solution analytique simple décrite par des équations algébriques de forme pure ;

- Les conditions de fin de phase (c'est-à-dire la description du développement de la plasticité dans le massif) lient le chargement mécanique au chargement thermique de façon extrêmement simple :

- La convergence de la galerie, définie comme le rapport entre le déplacement en paroi (positif vers

le centre) et le rayon, c'est-à-dire $U_{j} \equiv-\frac{u(a)}{a}$,

dépend des chargements thermiques $\theta$ et mécanique $\Delta \mathrm{P}$. Cette relation fonctionnelle, fortement non linéaire, peut se mettre sous la forme $\mathrm{EUi}=\mathscr{F}(\theta, \Delta \mathrm{P})$ pour toutes les phases traitées. La pertinence de la température caractéristique $\mathrm{T}^{*}=2 \mathrm{C}(1-v) / \mathrm{E} \alpha$, paramètre intrinsèque du matériau, est ainsi mise en évidence : un matériau dont la valeur de T* est deux fois plus grande que celle d'un autre peut supporter une température deux fois plus élevée, sans donner lieu à un supplément de déplacement.

\section{2}

\section{Concepts de trajet de chargement et de surface de convergence}

Pour un type de chargement thermique donné (par exemple flux constant imposé en paroi), les hypothèses (H2) et (H3) indiquent qu'il existe, à chaque instant donné, une correspondance biunivoque entre la température à la paroi $\theta$ (a) et la température $\theta(r)$ en un point quelconque du massif. Par conséquent, le chargement thermique peut être défini par la seule connaissance de cette température $\theta$ (a).

Cette propriété intéressante suggère de présenter le trajet de chargement (mécanique et thermique) sur le diagramme $(\Delta \mathrm{P}, \theta(\mathrm{a}))$ de la figure 7 (dans cette illustration on remplace le champ $\theta(\mathrm{r})$ par le scalaire $\theta(\mathrm{a})$ ).

Il apparaît, d'après une analyse détaillée présentée dans (Wong, 1992) que ce " plan de chargement » peut être partitionné en quatre zones distinctes (d'après les trois relations (11), (12), et (15)), correspondant chacune à une succession déterminée de zones plastiques et élastique.

On retrouve immédiatement les points soulignés précédemment, par exemple :

- en condition isotherme, on reste sur l'axe $\Delta \mathrm{P}$, et la configuration $\mathrm{RA}_{2} \mathrm{RF}_{2}-\mathrm{EL}$ de la phase 4 ne peut intervenir ;

- pour le cas de l'incompressibilité $v=0,5$, la ligne séparant les deux configurations $\mathrm{RF}_{1}$-EL et $\mathrm{RA}-\mathrm{RF} \mathrm{F}_{1}-\mathrm{EL}$ est horizontale, on ne peut donc pas provoquer l'apparition du régime d'arête par un chargement purement mécanique $(\theta=0)$.

Dans cette représentation, la séquence du chargement : d'abord une baisse de la pression interne, suivie par un échauffement en gardant la pression interne constante, n'est qu'un cas particulier qui correspond à un (ctrajet) d'abord horizontal, puis vertical, désigné $\mathscr{L}$. Ce trajet de chargement engendre une évolution des zones élasto-plastiques exactement dans l'ordre que l'on a constaté pendant les phases 1 à 4 , comme illustré sur la figure 8 .

Ce trajet particulier peut maintenant être généralisé à un trajet plus complexe, comme celui désigné $\mathscr{L}^{\prime}$ ce qui constitue une justification supplémentaire de la construction du plan de chargement. Par exemple, le cas $\Delta \mathrm{P}>1$ à la fin du chargement mécanique, ou $\Delta \mathrm{P}>0$ pendant l'échauffement, sont parfaitement admissibles. Le modèle, quant à lui, reste valable si on ne se déplace que de gauche à droite, et de bas vers le haut, au regard des hypothèses ( $\mathrm{H}^{\prime}$ ') et (H3). A titre d'exemple, l'évolution des zones élasto-plastiques correspondant au trajet $\mathscr{L}^{\prime}$, est illustrée sur la figure 9.

Par ailleurs, le fait que la fonctionnelle $\mathrm{EU}_{i}=\mathscr{F} \theta(\mathrm{r})$, $\Delta \mathrm{P})$ ne fait intervenir que les valeurs de ses arguments à l'instant actuel, et non pas leur histoire de l'évolution, est un résultat remarquable. Ceci signifie que, à condition que les hypothèses $(\mathrm{H} 1)$, (H2), et (H3) soient vérifiées, l'équilibre attteint à un instant quelconque ne dépend alors que de l'état du chargement au même instant ; il est indépendant du trajet suivi (Wong, 1994). Cette constatation permet d'associer une valeur unique 


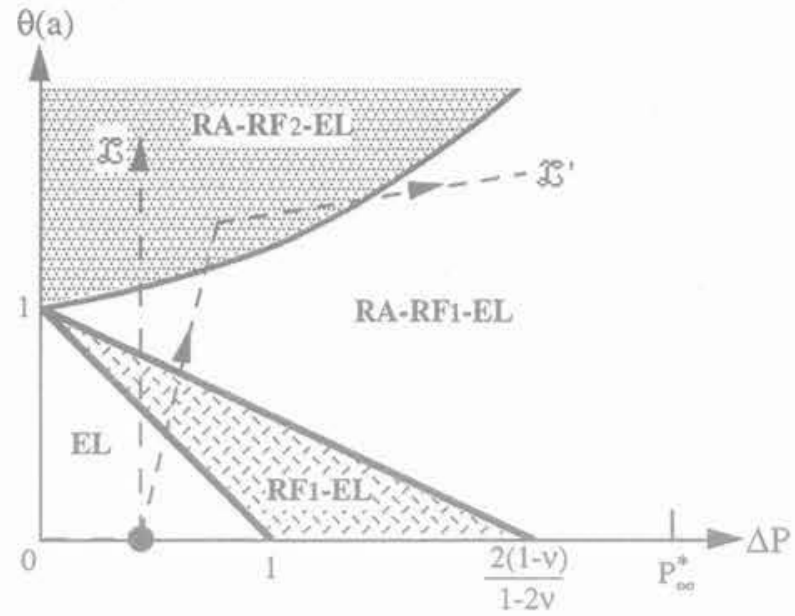

FIG. 7 Le plan de chargement, la partition en sous-domaines et les trajets de chargement.

The loading plane, the partition into subdomains and the loading paths.

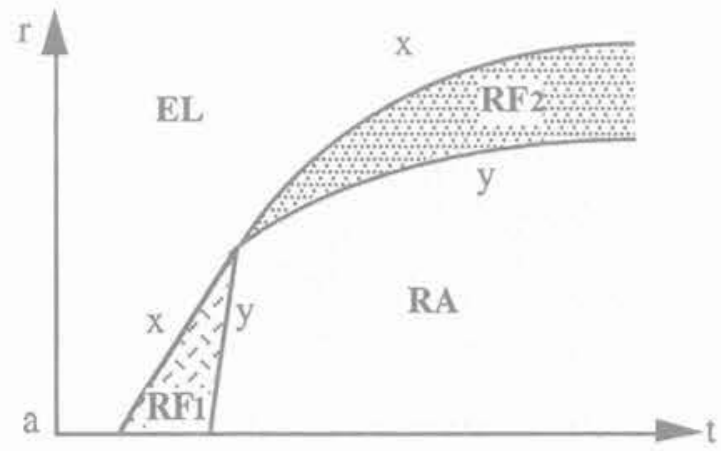

FIG. 8

Évolution des zones élasto-plastiques correspondant au trajet de chargement $X$. Evolution of the elastoplastic zones corresponding to the loading path 8 ,

de la convergence à chaque point du plan $(\Delta \mathrm{P}, \theta(\mathrm{a}))$, et de construire ainsi une " surface de convergence», comme illustrée par la figure 10. Celle-ci peut être considérée comme une généralisation au cas non isotherme du concept « courbe de convergence» en condition isotherme.

\section{Applications numériques}

\section{Exemple 1 : comparaison avec le code numérique " thyme "}

\section{1}

\section{Le programme thyme}

Dans ce premier exemple, on cherche à comparer les résultats numériques du modèle analytique, et ceux
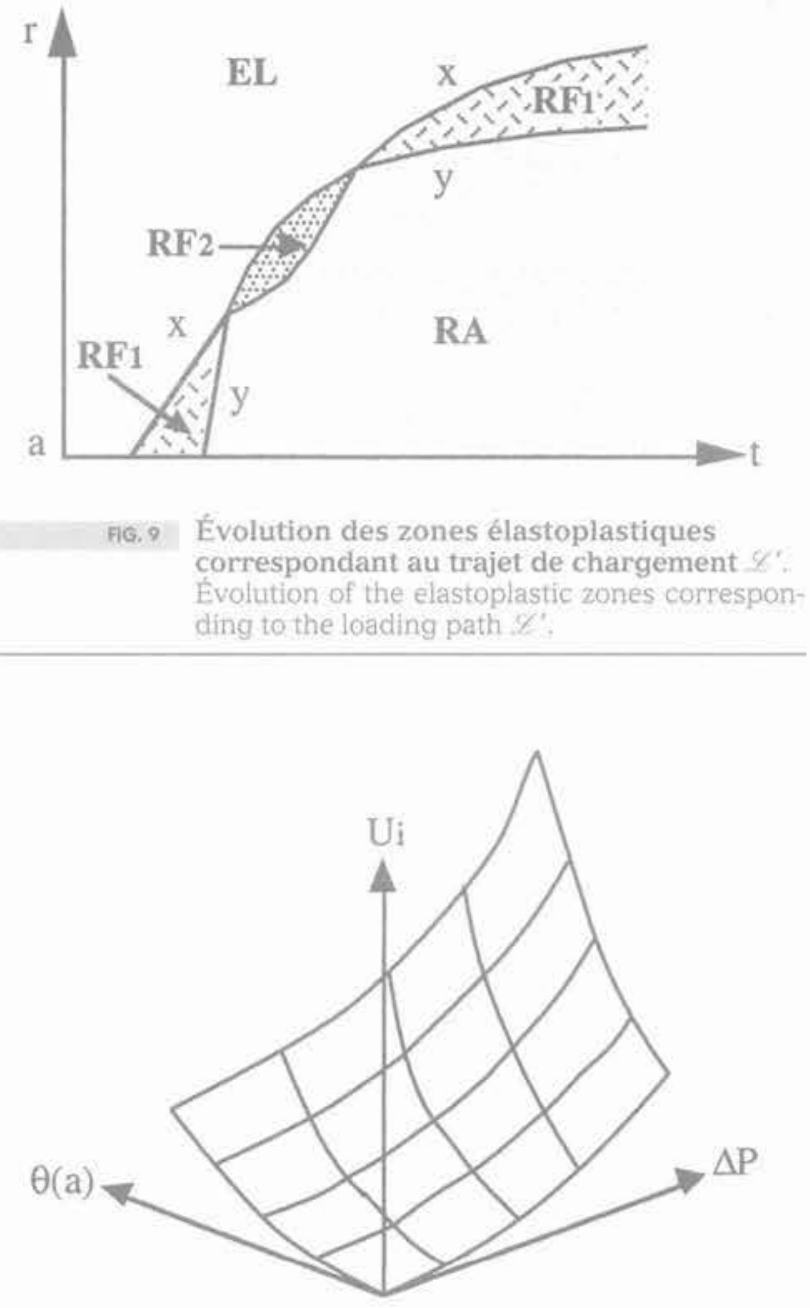

FG. 10 Le concept de la surface de convergence faisant intervenir le chargement thermique.

The concept of the surface of convergence including thermal loading.

issus d'un code de calcul numérique aux éléments finis. Cette comparaison permet de vérifier le bon fonctionnement du programme informatique du modèle analytique d'une part, et d'ajuster les paramètres de tolérance numérique du code THYME en présence du chargement thermique d'autre part.

Le programme THYME, développé par A. Giraud (Giraud, 1993) dans un cadre théorique plus général, traite des problèmes thermo-élasto-viscoplastiques et thermo-élasto-plastiques unidimensionnels des milieux poreux. La discrétisation spatiale se fait au moyen des éléments linéiques hermitiques (fonctions d'interpolation cubiques) qui assurent en chaque nceud la continuité ${ }^{C^{1}}$ du déplacement, et donnent une meilleure précision par rapport à d'autres types d'éléments qui n'assurent qu'une continuité $\mathrm{C}^{0}$

Dans l'étude comparative qui suit, le critère de Tresca est pris en compte, conformément à l'exigence du modèle analytique. Par contre, en raison de la nature du calcul numérique, l'état de contrainte aux points considérés (points de Gauss) ne se situe jamais sur une arête. Dans une zone où cela devrait théoriquement se produire, l'état de contrainte oscille entre deux 
faces adjacentes à des pas de temps successifs, et l'on reproduit numériquement le phénomène du régime d'arête.

\section{8}

\section{Configuration et paramètres de caicul}

Considérons le problème d'un forage de $0,5 \mathrm{~m}$ de diamètre soumis à un échauffement; la forme du chargement thermique considérếe est approximativement celle des déchets radioactifs de catégorie C (ANDRA, 1989). L'origine du temps $t=0$ est fixée arbitrairement au début de l'échauffement par commodité. Les divers paramètres pris en compte sont résumés dans le tableau I.

Il convient de remarquer que le flux de chaleur pris en compte correspond à une puissance volumique initiale de $6100 \mathrm{Wm}^{-3}$ soit deux fois plus élevée que celle des déchets radioactifs de type $\mathrm{C}$ après trente ans de préconditionnement au moment de la mise en place.
De plus, dans nos calculs, la longueur de la source thermique est infinie. Les deux hypothèses conduisent à une température maximale artificiellement élevée, de $460^{\circ} \mathrm{C}$ au bout de 70 mois (l'instant de paroxysme), et ne reflète donc pas les valeurs habituellement rencontrées en pratique $\left(100^{\circ} \mathrm{C}\right.$ à $\left.200^{\circ} \mathrm{C}\right)$. Ces valeurs élevées de la température ont été choisies volontairement dans le but de montrer que le modèle analytique reste applicable même à des températures très supérieures aux valeurs réalistes.

A partir des paramètres physiques du tableau $\mathrm{I}$, on déduit le chargement mécanique adimensionnel, ainsi que la température et le rayon caractéristique :

$$
\begin{gathered}
\Delta \mathrm{P}^{*} \equiv \frac{\mathrm{P}_{\infty}-\mathrm{P}_{1}}{\mathrm{C}}=0,5 ; \mathrm{T}^{*} \equiv \frac{2 \mathrm{C}(1-v)}{\mathrm{E} \alpha}=188^{\circ} \mathrm{C} \\
\frac{\mathrm{r}^{*}}{\mathrm{a}} \equiv \sqrt{\mathrm{e}^{\Delta \mathrm{P}^{*}}}=1,28
\end{gathered}
$$

Les calculs mécaniques sont menés jusqu'à 60 mois, l'hypothèse fondamentale $\partial_{t} \theta>0$ n'étant plus vérifiée après l'instant du paroxysme.

\begin{tabular}{|c|c|c|c|}
\hline \multicolumn{2}{|c|}{ Paramètres thermiques } & \multicolumn{2}{|c|}{ Paramètres mécaniques: } \\
\hline Conductivité & $\mathrm{K}=1,5 \mathrm{Wm}^{-1}{ }^{\circ} \mathrm{C}^{-1}$ & Module d'Young & $\mathrm{E}=3200 \mathrm{MPa}$ \\
\hline $\begin{array}{l}\text { Capacité calorifique } \\
\text { volumique }\end{array}$ & $C_{v}=2,910^{-6} \mathrm{Jm}^{-3}{ }^{\circ} \mathrm{C}^{-1}$ & Cohésion & $\mathrm{C}=6 \mathrm{MPa}$ \\
\hline Diffusivité $\left(\frac{\mathrm{K}}{\mathrm{C}_{\mathrm{v}}}\right)$ & $\mathrm{k}=0,5210^{-6} \mathrm{~m}^{2} \mathrm{~s}^{-1}$ & $\begin{array}{l}\text { Dilatation thermique } \\
\text { linéique }\end{array}$ & $\alpha=10^{-5}{ }^{\circ} \mathrm{C}^{-1}$ \\
\hline Flux exponentiellement & $\mathrm{Q}_{0}=1200 \mathrm{Wm}^{-1}$ & Coefficient de Poisson & $y=0,5$ \\
\hline $\begin{array}{l}\text { décroissant en paroi } \\
Q(t)=Q e^{-m}\end{array}$ & $\begin{array}{l}\omega=0,0231 \mathrm{an}^{-1} \\
\text { (demi vie } T_{n}=30 \text { ans) }\end{array}$ & Pression géostatique & $\mathrm{P}_{-}=8 \mathrm{MPa}$ \\
\hline & & Pression à la paroi & $\mathrm{P}_{1}=5 \mathrm{MPa}$ \\
\hline
\end{tabular}

TABIEAUT Paramètres thermomécaniques pris en compte dans le calcul comparatif.

- Les paramètres mécaniques E, C et $\alpha$ ci-dessus correspondent par exemple à celles de l'argile de l'Aisne (Rousset, 1988).
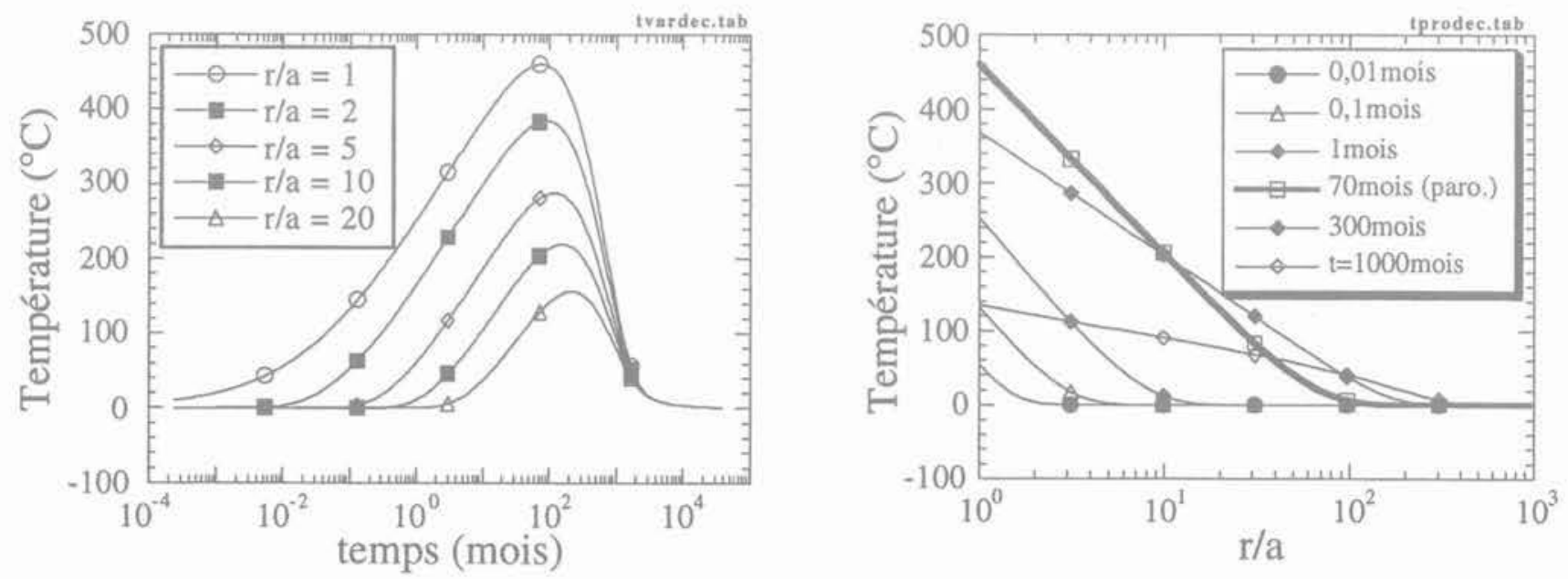

FiG. 11 Variation de température prise en compte dans l'étude comparative. Temperature variation considered in the comparative study. 


\section{1 .3}

\section{Champ thermique engendré}

Les variations de température d'après les données du dernier paragraphe sont illustrées sur la figure 11: elles ont une forme classique. On observe, comme mentionné précédemment, une valeur maximale de $460^{\circ} \mathrm{C}$, atteinte à la paroi au bout de 70 mois environ (temps de paroxysme).

\section{1 .4}

\section{Comparaison des résultats}

\section{Convergence en paroi}

Les convergences de la galerie en fonction du temps, obtenue d'après les deux méthodes de calcul, sont comparées sur la figure 12.

Un écart maximal de $0,2 \%$ est atteint au bout de 60 mois, une valeur négligeable en pratique. Remarquons toutefois que cette concordance est obtenue au prix d'un critère de convergence relativement sévère

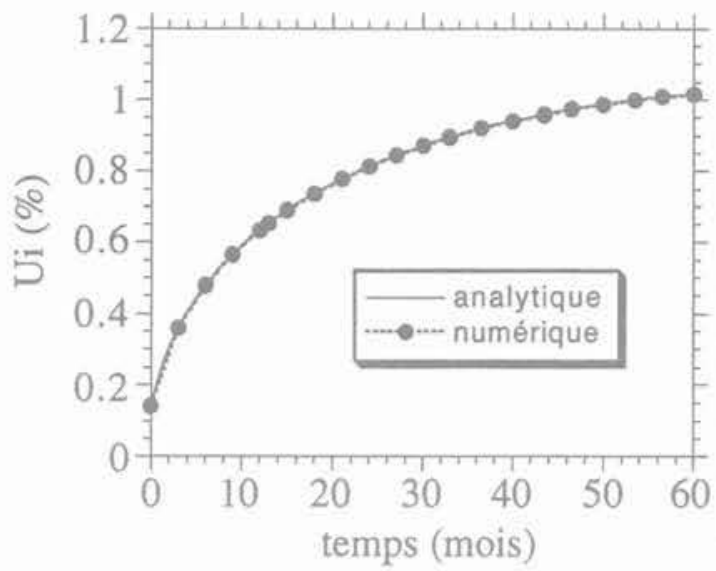

FIG. 12 Comparaison de la convergence d'après le modèle analytique et la méthode des éléments finis (trajet de type $\varnothing$ ).

Comparaison of the convergence between the analytical model and the finite element method (loading path of type 5 ). dans l'approche numérique. Pour des applications pratiques, il est légitime de les relaxer afin de raccourcir le temps de calcul.

\section{Profils des contraintes}

Les profils des contraintes à divers instants $(t=0$; 0,$25 ; 0,5 ; 1$ et 25 mois respectivement) sont tracés sur la figure 13. Les deux méthodes de calcul conduisent à des évolutions de contraintes quasi identiques ; leurs écarts ne sont pas visibles.

\section{7 .5 \\ Commentaires}

Si les deux approches donnent des résultats quasi identiques, c'est au niveau du temps de calcul que l'intérêt du modèle analytique est pleinement justifié ; ce dernier donne en effet des résultats quasiment instantanément, La sauvegarde des résultats, souvent très volumineux, est donc rendue inutile. Par conséquent, la gestion des informations est grandement simplifiée, ce qui constitue un avantage supplémentaire.

\section{2}

\section{Exemple 2 : étude comparative d'un forage dans quatre matériaux différents}

\section{2 .1 \\ Configurations et paramètres de calcul pris en compte}

Nous nous intéressons ici à la réponse mécanique d'un forage réalisé dans quatre types de matériaux différents. La géométrie, les paramètres thermiques ainsi que les chargements mécanique et thermique sont les mêmes que dans l'exemple précédent; seuls les paramètres mécaniques diffèrent d'un matériau à l'autre, et sont résumés dans le tableau II.

Retenons tout de suite les observations suivantes avant de commenter les résultats de calcul :

- pour les matériaux (2), (3), et (4), le massif est entière-

TABLEAU II Propriétés mécaniques des quatre types de matériaux pris en compte.

\begin{tabular}{|c|c|c|c|c|c|c|}
\hline \multirow[b]{2}{*}{ Matériaux } & \multicolumn{3}{|c|}{ Paramètres physiques } & \multicolumn{3}{|c|}{ Paramètres adimensionnels } \\
\hline & $\underset{(\mathrm{MPa})}{E}$ & $\underset{(\mathrm{MPa})}{\mathrm{C}}$ & $\stackrel{\alpha}{\left({ }^{\circ} \mathrm{C}^{-1}\right)}$ & $\Delta \mathrm{P}^{*} \equiv \frac{\mathrm{P}_{-}-\mathrm{P}}{\mathrm{C}}$ & $T^{*} \equiv \frac{2 C(1-v)}{E \alpha}$ & $E^{*} \equiv \frac{E}{C}$ \\
\hline (1) Argile de Boom & 230 & 1,3 & $10^{-5}$ & 2,3 & 565 & 177 \\
\hline (2) Argile de Lacq & 2500 & 10,0 & $10^{-5}$ & 0,3 & 400 & 250 \\
\hline (3) Argile de l'Aisne & 3200 & 6,0 & $10^{-5}$ & 0,5 & 188 & 533 \\
\hline (4) Granite & 68000 & 70,0 & $7.10^{-6}$ & 0,04 & 147 & 971 \\
\hline
\end{tabular}

Voir (Rousset, 1988), (Bérest et al., 1985) et (Djéran, 1992). 

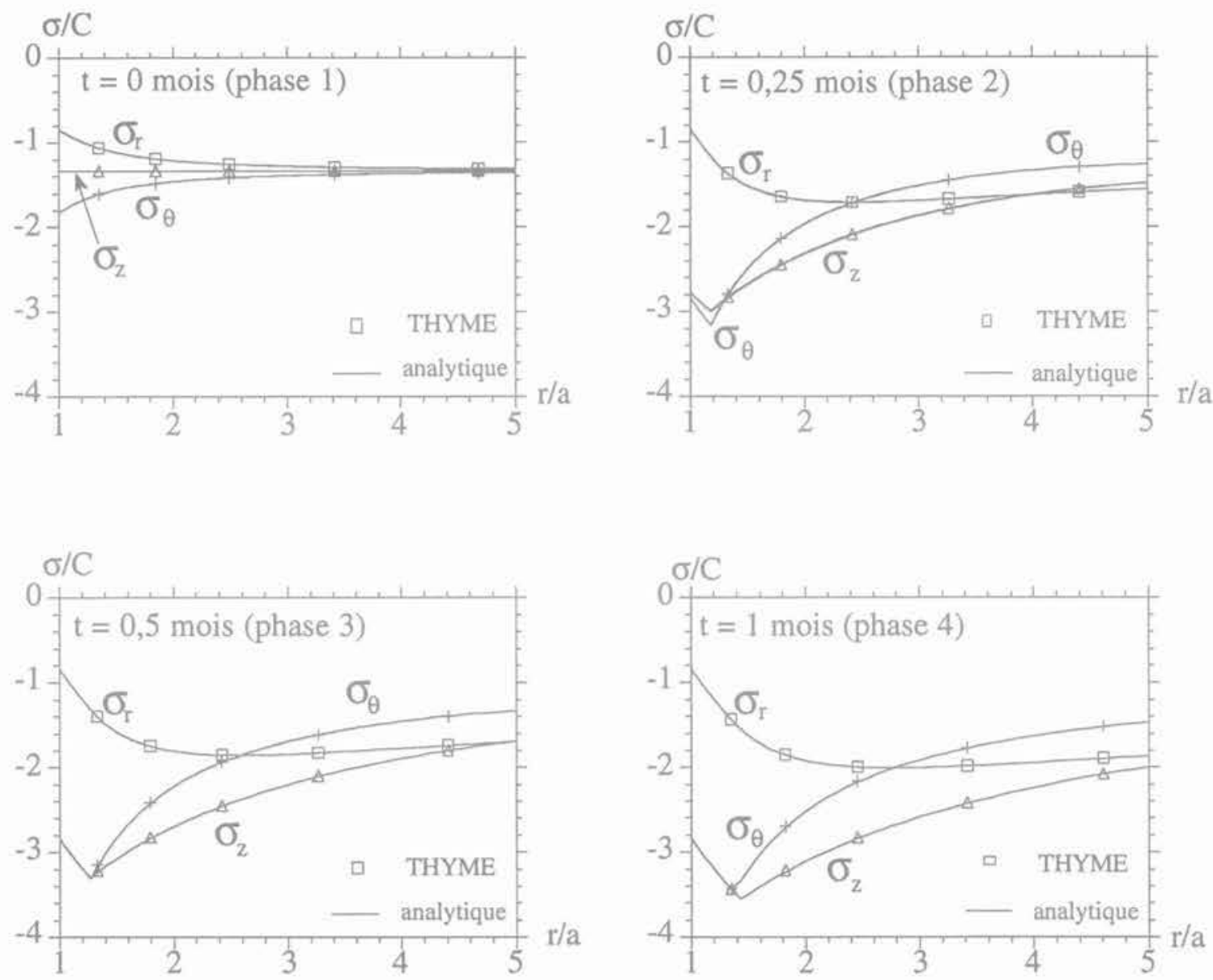

FG. 13 Comparaison des profils de contraintes à divers instants entre le modèle analytique et la méthode numérique.

Comparaison of stress profiles at various instants between the analytical model and finite element method.

ment élastique avant l'échauffement $\left(\Delta \mathrm{P}^{*}<1\right)$. Par contre, dans le cas de l'argile de Boom, la plastification acquise pendant la phase de chargement mécanique est significative $\left(\Delta \mathrm{P}^{*}>1\right)$;

- toujours dans le cas de l'argile de Boom, compte tenu du rapport entre la température maximale atteinte en paroi $\left(460^{\circ} \mathrm{C}\right)$ et la température caractéristique $\left(565^{\circ} \mathrm{C}\right)$, on traverse uniquement les phases 1 et 2 , on ne voit donc pas l'apparition de la zone en régime d'arête; - l'applicabilité du critère de Tresca au cas du granite est évidemment discutable, on vise ici surtout à dégager les caractéristiques du modèle, par un jeu de paramètres aussi variés que possible.

\section{9}

\section{Résultats et commentaires}

Sur les figures 14 et 15 , sont tracées respectivement les variations de la convergence et de l'extension de la zone plastique en fonction du temps.

Visiblement, J'extension de la zone plastique, et surtout la convergence, provoquées par le chargement thermique peuvent atteindre le même ordre de grandeur que celles dues au chargement mécanique, voire même les dépasser largement en cas de chargement thermique intensif, A titre d'exemple, l'argile de l'Aisne présente une convergence totale de $1 \%$ à 60 mois, soit 7 fois la convergence initiale due au creusement. Pour les argiles de Boom et de Lacg, on aboutit respectivement à $5,6 \%$ et $0,2 \%$ de convergence au mème instant, soit 1,8 et 1,3 fois les convergences initiales correspondantes.

En toute logique, la convergence maximale apparaît dans le cas de l'argile de Boom, dont la rigidité et la résistance sont les plus faibles. En revanche, la comparaison entre les trois matériaux (2), (3) et (4) est plus confuse. Dans le granite, qui présente la plus faible convergence au départ (inversement proportionnelle au module E en élasticité), la convergence dépasse celle de l'argile de Lacq au cours de l'échauffement, mais reste inférieure à celle de l'argile de l'Aisne.

Paradoxalement, l'argile de Lacq présente la convergence totale la plus faible, bien qu'aucun de ces parametres thermomécaniques $T^{*}, E^{*}$, et $\Delta \mathrm{P}^{*}$ ne soit extrémal.

Ce paradoxe ne sera levé que par l'étude paramétrique ci-après.

\section{2 .3}

\section{Étude paramétrique autour de l'argile de Lacq}

Le paradoxe posé par l'argile de Lacq est en fait une indication précieuse pour mieux comprendre le méca- 


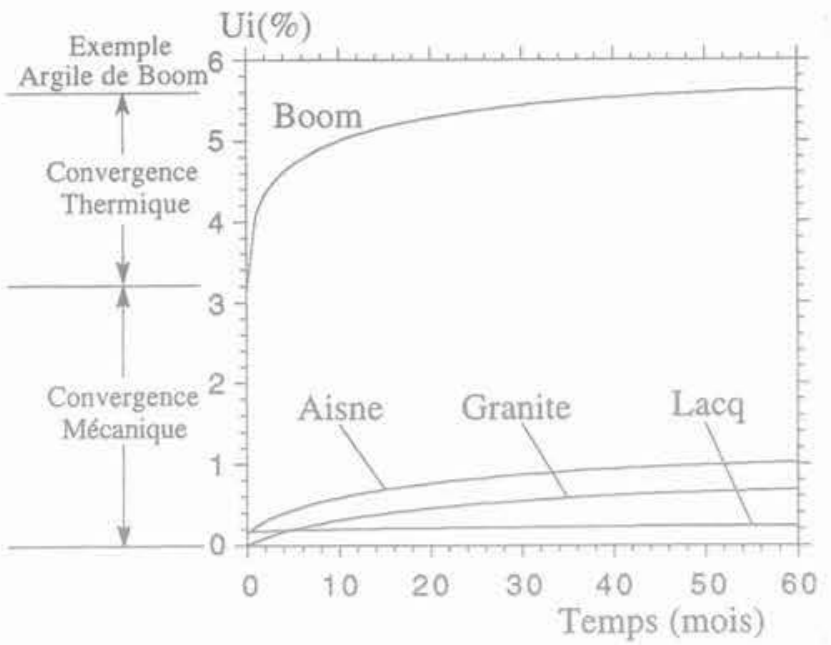

F19. 14 Evolution de la convergence. Evolution of the convergence.

nisme du couplage thermomécanique dans le cas d'un matériau élasto-plastique parfait.

Pour lever ce paradoxe, on se propose de réaliser une étude paramétrique restreinte autour des paramètres de l'argile de Lacq, qui est donc considéré dans ce qui suit comme le cas de référence.

\section{Influence du chargement mécanique $\Delta \mathrm{P}^{*}$}

Sur la figure 16 sont tracées les variations de la convergence en fonction du temps d'échauffement pour diverses valeurs de P maintenues constantes. Bien évidemment, la convergence est une fonction décroissante de $\mathrm{P}$.

En revanche, il convient de remarquer l'influence importante du chargement mécanique sur la convergence provoquée par l'échauffement.

A titre d'indication, la part de convergence due exclusivement à l'échauffement au bout de 60 mois passe de $0,22 \%$ pour $\mathrm{P}_{\mathrm{t}}=1,5 \mathrm{MPa}$, à $0,08 \%$ et $0,01 \%$ pour $\mathrm{P}_{1}=4,5 \mathrm{MPa}$ et $\mathrm{P}_{4}=7,5 \mathrm{MPa}$ respectivement.

Par ailleurs, conformément aux prédictions du

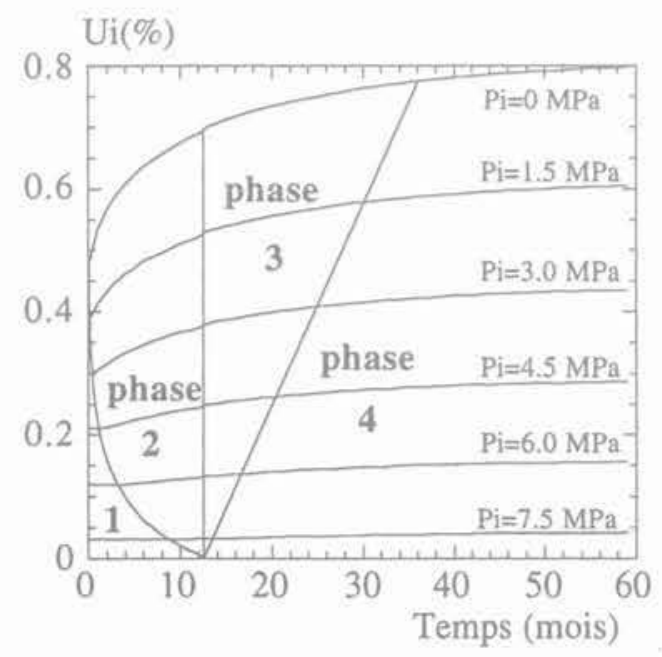

FG. 16 Influence du chargement mécanique. Influence of mechanical loading.

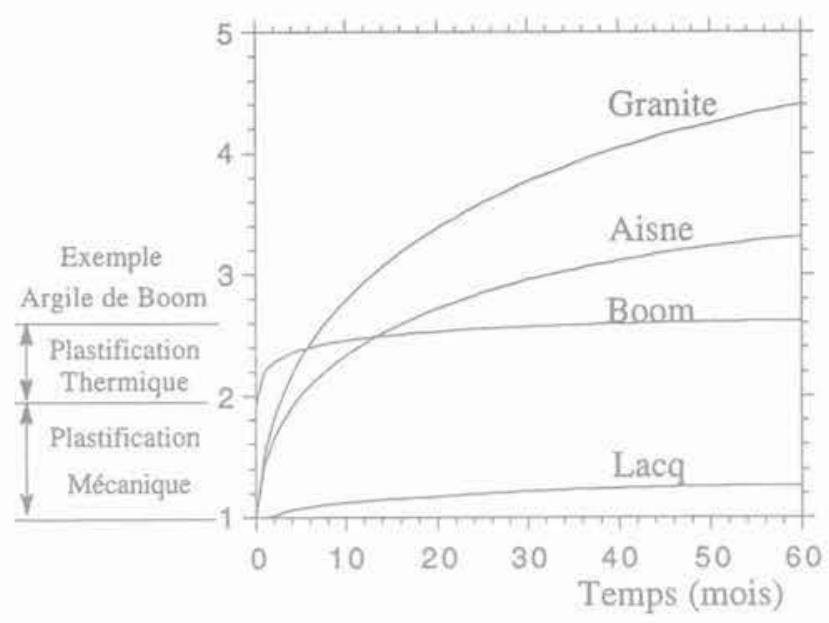

FlG. 15 Evolution de la frontière plastique. Evolution of the plastic front.

modèle, la phase élastique est d'autant plus longue que $P$ est grand; la verticalité de la ligne séparant les phases 2 et 3 est, quant à elle, une conséquence directe de l'hypothèse $v=0,5$ (car le pivot $B$ de la figure 4 est alors indépendant de $\left.\Delta \mathrm{P}^{*}\right)$. Le cas particulier $\mathrm{P}_{i}=\mathrm{P}_{\infty}\left(\Delta \mathrm{P}^{*}\right.$ $=0$ ), conduisant à un écrasement des phases 2 et 3 , est également traité par le modèle.

\section{Influence du module d'Young $\mathrm{E}$}

D'un point de vue physique, une étude paramétrique faisant varier uniquement la valeur de E pourrait être sujette à discussion. Car, en réalité, un matériau possédant un module d'Young plus fort a, dans la plupart des cas, une cohésion $C$ plus élevée. Il n'en reste pas moins que le rapport $\frac{\mathrm{E}}{\mathrm{C}}$ peut effectivement varier d'un géomatériau à l'autre, ce que le tableau II a clairement mis en évidence. Cette étude présente donc les indications utiles à la compréhension du couplage thermomécanique.

Soulignons que le module d'Young joue un double rôle : c'est d'abord la rigidité du matériau qui tend à réduire les déplacements, mais en même temps, c'est aussi la constante de proportionnalité de la contrainte thermique $\mathrm{E} \alpha \mathrm{T}$. Cette relation à double facette est clairement exprimée par la relation fonctionnel-

le $\frac{E}{C} U_{1}=F\left(\frac{E \alpha T}{2 C(1-v)} ; \frac{P_{\infty}-P_{i}}{C}\right)$, qui résume l'analyse des quatre premières phases (cf. expressions (A4), (A12), (A17), et (A20)). Par conséquent, le sens de variation de la convergence, entraînée par une augmentation de E, n'est pas a priori évident.

Sur la figure 17, sont tracées les variations de la convergence $U_{1}$ en fonction du temps, pour diverses valeurs du module d'Young E. En condition isotherme $(\mathrm{a} t=0)$, la convergence diminue pour une augmentation de E: c'est un résultat tout à fait classique. Par contre, la part de la convergence provoquée par l'échauffement (appelons-la convergence thermique) présente une tendance exactement inverse : à l'exception de la phase élastique, pendant laquelle la convergence thermique est nulle, elle est d'autant plus importante que la valeur de E est élevée. 


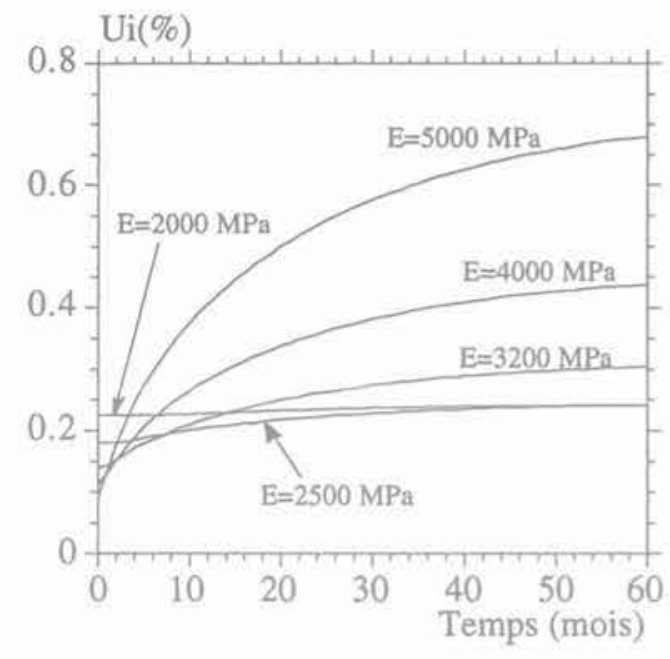

FG. 17 Influence du module d'Young. Influence of Young's modulus.
La relation entre la convergence totale et le module d'Young dépend donc des intensités relatives des chargements thermique et mécanique.

C'est cette dernière observation qui permet d'expliquer le paradoxe soulevé par l'argile de Lacq. Les trois matériaux (2), (3), et (4) du tableau étant élastiques au départ $\left(\Delta \mathrm{P}^{*}<1\right)$, leurs convergences initiales sont alors inversement proportionnelles à leurs modules d'Young, tandis que l'argile de Boom conduit à la convergence initiale « à froid » de loin la plus importante, en raison de la plastification initiale signficative $\left(\Delta \mathrm{P}^{*}>1\right)$. Une fois que le massif est entré en plasticité, la forte valeur de $T^{*}$ (inversement proportionnelle à E) dans le cas de l'argile de Lacq tend à réduire l'importance du chargement thermique $\theta$ par rapport à d'autres matériaux. pour une même élévation de la température, de telle sorte qu'au-delà d'un certain seuil thermique, sa convergence repasse en dessous de celle de l'argile de l'Aisne.

Dans la fourchette de températures rencontrées, la forte convergence initiale de l'argile de Boom n'est pas rattrapée par les autres matériaux.

\section{Influence du coefficient de dilatation thermique linéique $\alpha$}

Comme $\alpha$ apparaît en tant qu'une constante de proportionnalité dans la définition de la perturbation thermomécanique $\theta$, doubler $\alpha$ équivaut à doubler en tout point du massif la température en gardant $\alpha$ constant (c'est en fait le produit $\alpha \mathrm{T}$ qui intervient dans le couplage thermomécanique).

La figure 18 présente l'évolution de la convergence au cours du temps pour diverses valeurs de $\alpha$, où l'on observe que l'influence de ce dernier est singulièrement importante. A titre indicatif, la convergence thermique à 60 mois varie de $0,06 \%$ à $1,2 \%$, soit un rapport de 1 à 20 , quand $\alpha$ augmente de $10^{-5}{ }^{\circ} \mathrm{C}^{-1}$ à $2 \mathrm{x}$ $10^{-5}{ }^{\circ} \mathrm{C}^{-1}$. soit un rapport de 1 à 2 . Ceci montre que la fonctionnelle $\mathscr{F}(\theta(r), \Delta P)$ est fortement non linéaire.

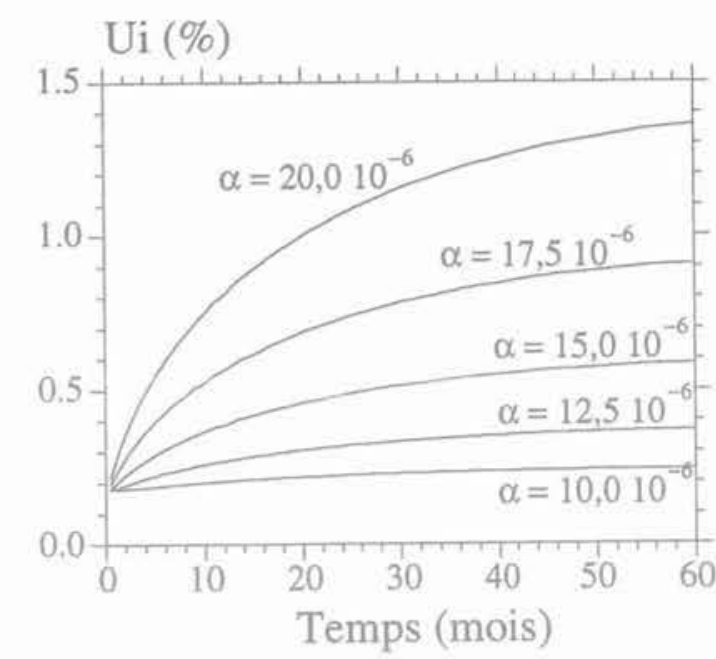

FG. 18 Influence du coefficient de dilatation thermique linéique.

Influence of the coefficient of linear thermal expansion.
Dans un calcul pratique, $\alpha$ peut dépendre de la température, et il faudrait dans ce cas choisir une valeur moyenne dans l'intervalle de température anticipé.

\section{9}

\section{Convergence-Confinement avec chargement thermique}

La solution originale, mise au point dans le cadre de cette recherche, incite à intégrer l'aspect thermique dans l'approche de dimensionnement simplifiée des ouvrages souterrains par la classique méthode Convergence-Confinement ( $\mathrm{CV}$-CF), cette dernière étant connue par un grand nombre d'ingénieurs praticiens.

Ce rapprochement présente néanmoins quelques difficultés, notamment en raison du caractère réparti du chargement thermique et de l'absence du régime permanent thermique en axisymétrie et milieu infini dans le cas du flux thermique imposé constant en paroi.

Toutefois, le cas particulier de la température imposée constante en paroi permet de contourner en partie cet obstacle, car, bien que le régime thermique permanent n'existe toujours pas, la convergence, en revanche, tend vers une valeur asymptotique $\mathrm{U}_{i}^{f}$ bien définie. Cette constatation permet de généraliser convenablement le concept de courbe de convergence à long terme au cas non isotherme, dans le cas particulier où la température est imposée constante en paroi.

Les résultats obtenus peuvent se résumer sous la forme d'une famille de courbes, et l'ensemble constitue une première approche pour tenir compte du chargement thermique dans la méthode CV-CF.

Dans les paragraphes qui suivent, après une discussion plus approfondie des difficultés de cette généralisation, on évalue la valeur de la convergence asympto- 
tique $\mathrm{U}_{i}^{f}$ en fonction de $\Delta \mathrm{P}^{*}$ et de la température en paroi $\theta_{a}$ (imposée constante). La pertinence du choix de $\mathrm{U}_{\mathrm{f}}^{\mathrm{f}}$ comme paramètre dimensionnant du problème est ensuite discutée en examinant l'évolution de la convergence en fonction du temps.

\section{- Les rayons plastiques}

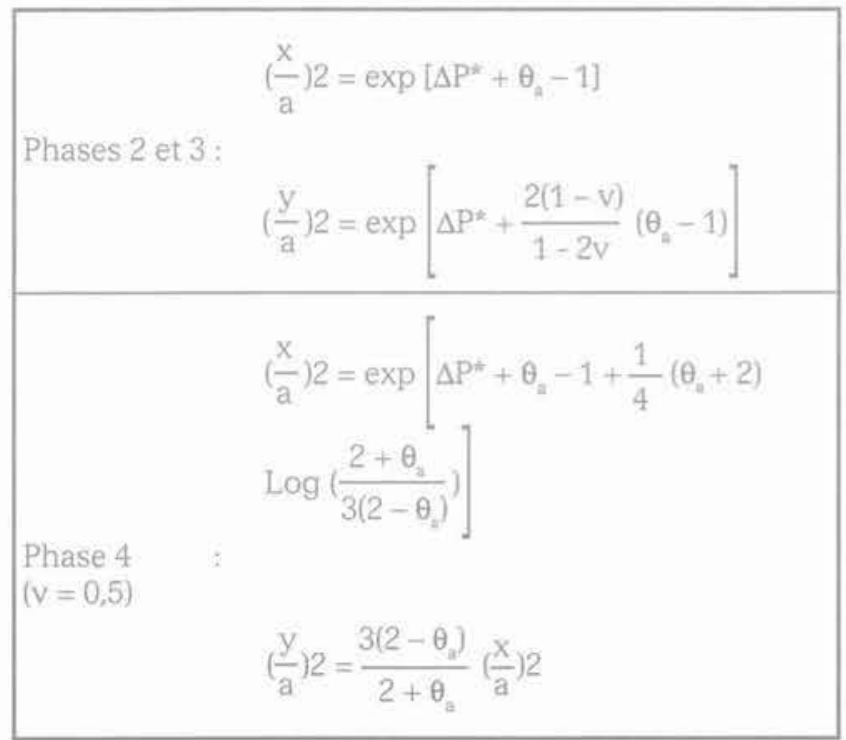

\section{1}

\section{Convergence asymptotique dans le cas particulier de la température imposée constante en paroi}

Le cas particulier de la température imposée constante en paroi $\left(\theta(\mathrm{a}, \mathrm{t})=\theta_{3}\right)$ admet une solution analytique explicite (paragraphe 3). La particularité de cette solution réside dans le fait que, malgré l'absence de régime permanent thermique, en ce sens que la norme $L_{2}$ :

$$
\left\|\theta(\mathrm{r}, \mathrm{t})-\theta_{\mathrm{a}}\right\|_{\frac{1}{2}} \equiv\left[\int_{\mathrm{a}}^{\infty}\left(\theta(\xi, \mathrm{t})-\theta_{\mathrm{a}}\right)^{2} \mathrm{~d} \xi\right]^{\frac{1}{2}}
$$

diverge lorsque $t \rightarrow \infty$, la température en tout rayon donné en revanche tend vers $\theta$ lorsque le temps $t$ tend vers l'infini, c'est-à-dire $\lim _{1 \rightarrow \infty}\left|\theta(\mathrm{r}, \mathrm{t})-\theta_{3}\right|=0$ pour tout $r$. En d'autres termes, la fonction $\theta(r, t)$ tend vers $\theta$ lorsque $t$ tend vers l'infini, mais de façon non uniforme.

En substituant $\theta(r)=\theta_{3}=$ constante $(<2)$ dans les équations qui déterminent les rayons plastiques, on obtient des solutions bien définies pour chacune des quatre phases décrites au paragraphe 6 :

Lorsque $\theta$ tend vers la valeur 2 , le rayon plastique $x$ tend vers l'infini, mais la convergence, quant à elle, tend vers une valeur finie donnée par 2 exp $\left(\Delta \mathrm{P}^{*}+1\right)-3$.

\section{- La convergence}

$$
\begin{aligned}
& \text { Phase 1: } \mathrm{E}^{*} \mathrm{U}_{\mathrm{f}}^{\mathrm{f}}=(1+v) \Delta \mathrm{P}^{*} \\
& \text { Phase 2: } E^{*} U_{j}^{f}=(1+v)\left\{2(1-v)\left[\begin{array}{l}
\exp \left(\Delta P^{*}\right. \\
\left.+\theta_{a}-1\right)-\theta_{a}
\end{array}\right]\right. \\
& \left.-(1-2 v) \Delta \mathrm{P}^{*}\right\} \\
& \text { Phase 3: } E^{*} U_{1}^{f}=2\left(1-v^{2}\right) \exp \left(\Delta P^{*}+\theta_{3}-1\right) \\
& \begin{array}{l}
+\frac{(1-2 v)^{2}}{2} \exp \left[\Delta \mathrm{P}^{*}+\frac{2(1-v)}{1-2 v}\left(\theta_{a}-1\right)\right] \\
+\frac{(1-2 v)}{2}\left(1-3 \Delta \mathrm{P}^{*}\right)-3(1-v) \theta_{a}
\end{array} \\
& \text { Phase 4: } \\
& (v=0,5)
\end{aligned}
$$

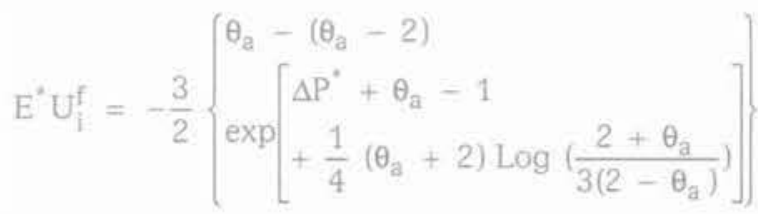

Ces résultats sont remarquables. Ils impliquent l'existence d'un régime permanent mécanique, dans le sens où la convergence en paroi tend vers une valeur finie au bout d'un temps infini (en pratique au bout d'un temps très long), alors qu'il n'y a pas de régime thermique permanent.

Les résuitats analogues n'existent pas pour le cas de flux imposé constant en paroi, car la température en tout point est alors sans cesse croissante et sans borne, ainsi que toutes les quantités mécaniques.

\section{2}

\section{Courbes de convergence en régime permanent mécanique}

Compte tenu des discussions précédentes, dans le cas où la température est imposée constante $\theta(a, t)=\theta_{\text {a }}$ en paroi, et au bout d'un temps infini, la convergence $\mathrm{U}(\mathrm{t})$ tend vers une valeur asymptotique $\mathrm{U}^{f}$ uniquement déterminée par les deux paramètres scalaires de chargement $\Delta \mathrm{P}$ et $\theta_{\text {. }}$. Cette relation définit une surface de convergence dans l'espace $\left(\mathrm{U}_{p}^{f}, \Delta \mathrm{P}, \theta_{\mathrm{a}}\right)$, dont l'allure a déjà été donnée sur la figure 10.

Une représentation qui se rapproche davantage de la méthode CV-CF est donnée sur la figure 19, en remplaçant l'axe classique $\mathrm{P}$, par $-\Delta \mathrm{P}^{*}$. Cette représentation permet de regrouper avantageusement sur le même graphique les courbes de convergence correspondant à différentes valeurs de $\mathrm{P}_{\alpha}$ car d'après notre modèle, c'est uniquement la différence $\mathrm{P}_{\alpha}-\mathrm{P}_{1}$ qui intervient. 

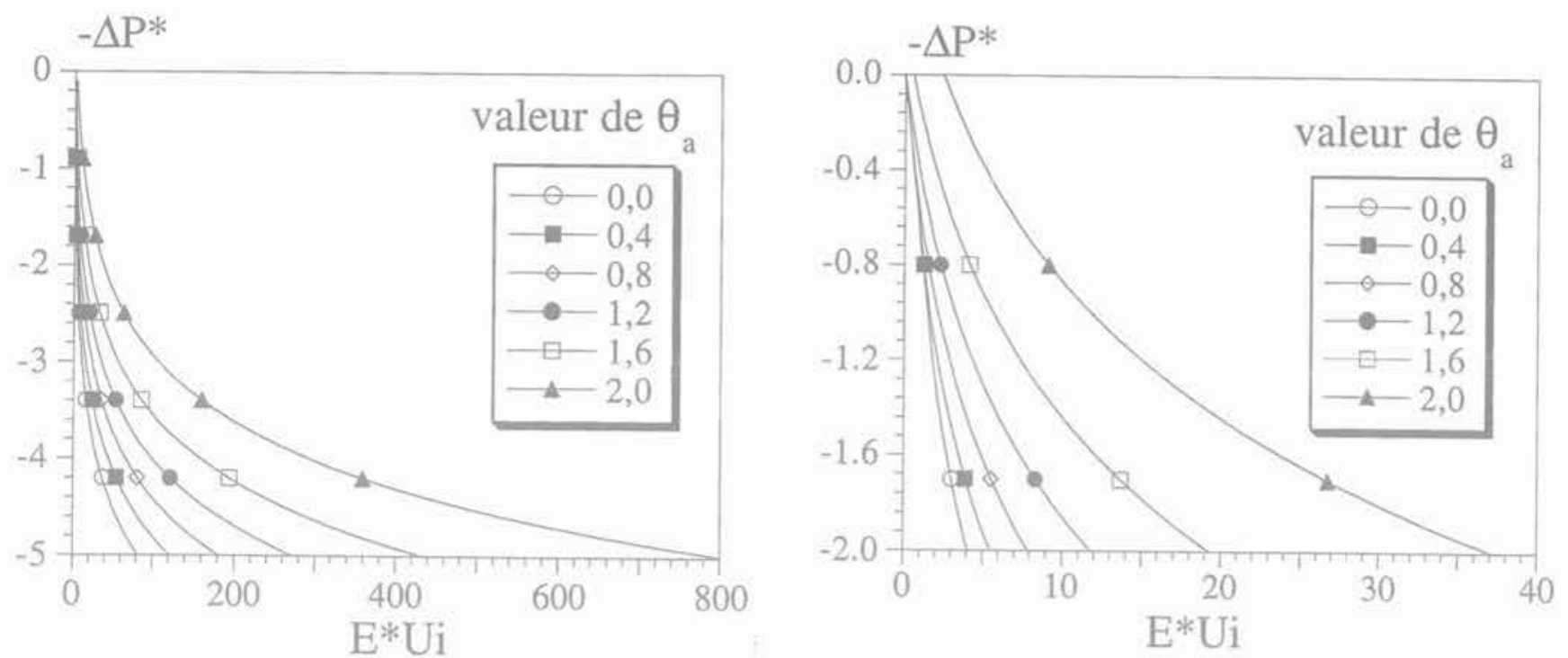

F6. 19 Courbes de convergence en régime permanent en présence d'un champ thermique.

Convergence curves at permanent state in the presence of a temperature field.

\section{3}

\section{Température imposée constante en paroi. Évolution de la convergence en régime thermique transitoire}

Ces valeurs de convergence Uf en régime permanent sont évidemment les valeurs majorantes des convergences pendant la phase transitoire. De façon à estimer quantitativement la pertinence physique de $U^{t}$, il paraît utile d'étudier le régime transitoire pour évaluer la constante de temps de la convergence. En d'autres termes, on s'intéresse à la vitesse à laquelle $\mathrm{U}(\mathrm{t})$ tend vers $U^{\text {? }}$.

Le champ thermique engendré par une température constante en paroi est classique. Cette évolution est illustrée sur la figure 20 ci-dessous.

Prenons arbitrairement $\Delta \mathrm{P}^{*}=2$, et considérons les trois cas de chargement thermique suivants :

$$
\theta_{\text {i }}=0,4 ; 0,8 ; 1,2
$$

Les figures 21 montrent l'évolution de $E^{*} U_{i}(t)$, puis du rapport $\frac{U_{i}(t)}{U_{i}^{f}}$ en fonction du temps adimensionnel $t^{\prime} \equiv \frac{\mathrm{kt}}{\mathrm{a}^{2}}$. Les résultats indiquent que, partant d'une même convergence initiale, le régime permanent mécanique est plus rapidement atteint lorsque l'échauffement est moins intense. Par exemple, pour atteindre $80 \%$ de la convergence finale, il faut un temps adimensionnel de $t^{\prime}=50$ pour $\Delta \mathrm{P}^{*}=2$ et $\theta_{\mathrm{a}}=1,2 ;$ tandis que pour la même valeur de $\Delta P^{*}$ et pour $\theta_{\mathrm{a}}=0,8$ et 0,4 , les durées adimensionnelles correspondantes seront réduites à 2,4 et 0,07 respectivement.

A titre d'indication, pour une galerie de rayon de $\mathrm{a}=1 \mathrm{~m}$, et un massif de diffusivité thermique de $\mathrm{k}=10^{-6} \mathrm{~m}^{2} \mathrm{~s}^{-1}$ (valeur moyenne représentative pour les géomatériaux), $\mathrm{t}^{\prime}=50$ correspond à un temps physique d'environ 19 mois et $t^{\prime}=2,4$ et 0,07 , à 1 mois et 19 heures respectivement.
Sachant qu'en pratique $\theta_{\text {a }}$ se situe plutôt en dessous de la valeur 1 (car $\mathrm{T}<\mathrm{T}^{*}$ en général), le régime permanent mécanique, du point de vue de la convergence, semble être assez rapidement atteint, de quelques heures à quelques mois (si l'on retient le critère de $80 \%$ de la convergence finale).

Ce résultat est important et justifie l'intérêt pratique de la valeur asymptotique $\mathrm{U}_{f}^{f}$ : dans les cas courants, $\mathrm{U}_{\mathrm{f}}^{t}$ représente effectivement la valeur de la convergence d'un ouvrage d'une durée de vie ordinaire en géotechnique.

\section{4}

\section{Exemple d'application. Étude de soutènement}

L'un des points intéressants de la méthode de Convergence-Confinement est la facilité avec laquelle on peut étudier l'interaction entre le massif et le soutènement, puis trouver le point d'équilibre. Lorsque l'effet de viscosité peut être négligé, ce dernier est uniquement déterminé par la courbe de convergence de la galerie, la courbe de confinement du soutènement, ainsi que la convergence $\mathrm{U}^{0}$ acquise au moment de la pose du soutènement.

Prenons le cas d'un soutènement élastique dont la courbe de confinement est une ligne droite, la figure 22 donne une illustration graphique de cette méthode.

Il convient de rappeler que la validité de la méthode repose notamment sur l'hypothèse d'un chargement monotone (décroissance monotone de la pression interne P), qui exclut toute décharge élastique en condition isotherme. Au cas où le massif est soumis à l'échauffement en présence d'un soutènement, des calculs non présentés ici montrent que, à cause de la présence du soutènement, la pression interne doit croître lorsque la température au sein du massif augmente, et on ne peut exclure la possibilité d'une décharge élastique. 

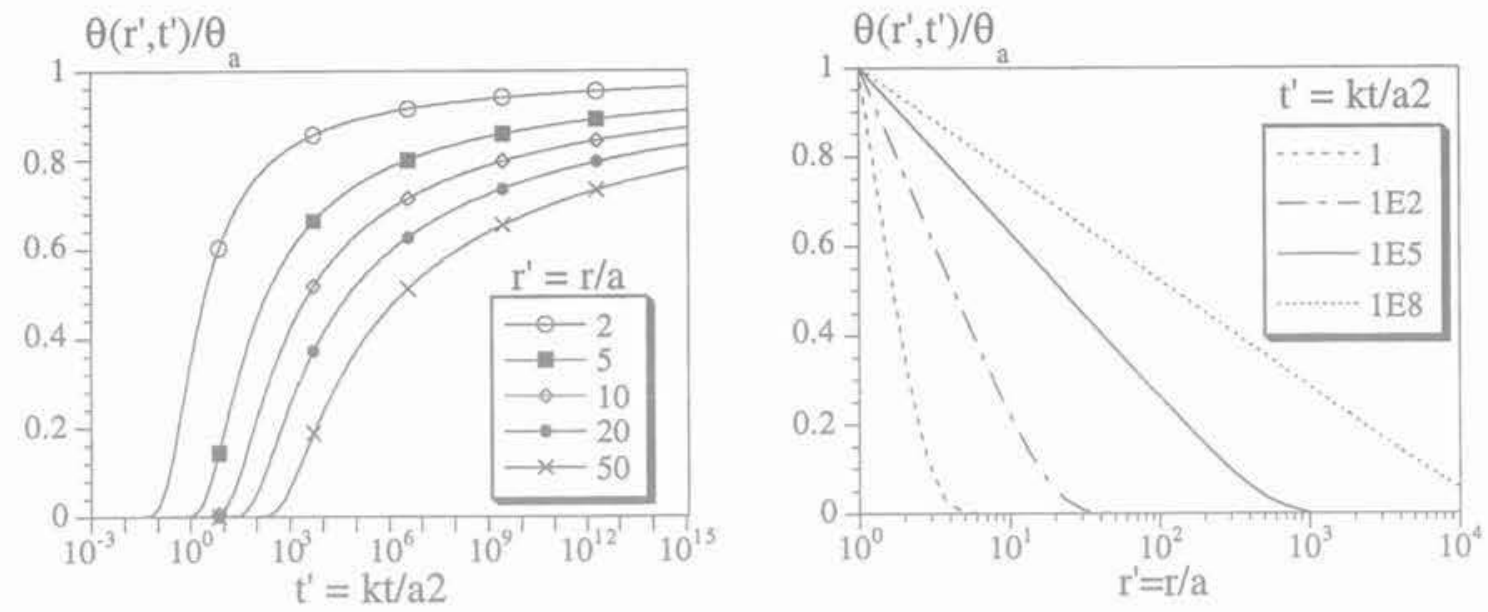

FG. 20 Evolution du champ thermique engendré par une température constante en paroí. Evolution of the temperature field generated by a constant temperature at the tunnel wall.
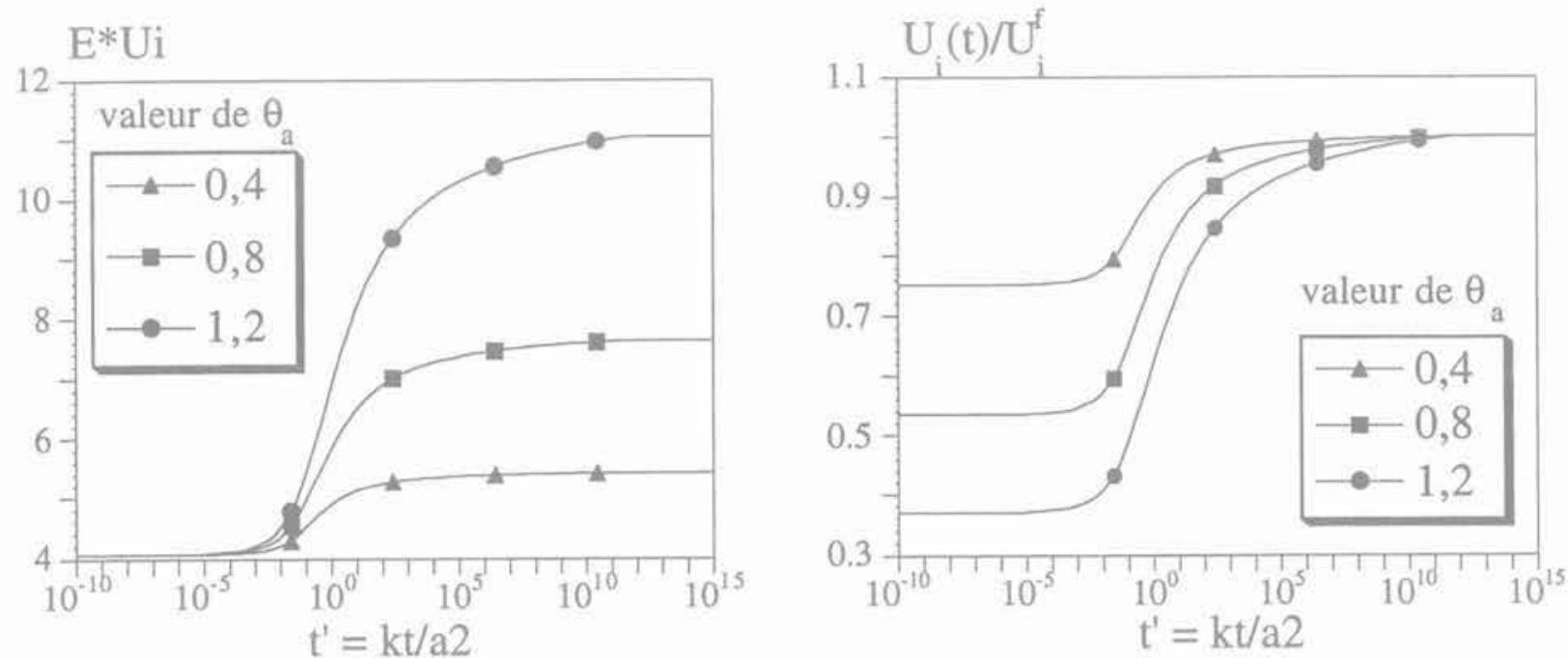
FG. 21 Variation de la convergence en fonction du temps adimensionnel $t^{\prime}=\frac{k t}{a^{2}}$ en régime thermique transitoire,
avec $\Delta \mathrm{P}^{*}=2$.

Variation of the convergence as a function of the dimensionless time $t^{*}=\frac{k t}{a^{2}}$ during the transient thermal state, with $\Delta \mathrm{P}^{*}=2$.

Toutefois, dans le cadre d'une préétude, si l'on souhaite connaitre l'ordre de grandeur des paramètres, notamment $\mathrm{U}$ et $\mathrm{P}$ à l'état d'équilibre, la méthode graphique décrite ci-dessus pourrait fournir une valeur approchée, faisant abstraction du phénomène de décharge élastique. Par ailleurs, dans le cas particulier où $P$ n'évolue pas beaucoup pendant le chargement thermique (cas des cintres coulissants par exemple), la solution proposée ici est exacte.

Dans ce cas, pour qu'on puisse utiliser l'abaque présenté, on doit effectuer un changement de variables et suivre la procédure décrite ci-dessous.

1. Déterminer $\mathrm{P}_{\infty}^{*} \equiv \frac{\mathrm{P}_{\infty}}{\mathrm{C}}, \mathrm{E}^{*} \equiv \frac{\mathrm{E}}{\mathrm{C}}$, et $\mathrm{U}_{\mathrm{i}}$ (convergence acquise à l'instant de pose) ;

2. Tracer une ligne horizontale à la hauteur de $-\Delta \mathrm{P}^{*}=-\mathrm{P}_{\infty}^{*}$ (qui correspond à l'axe horizontal de l'abscisse de la Fig. 22);

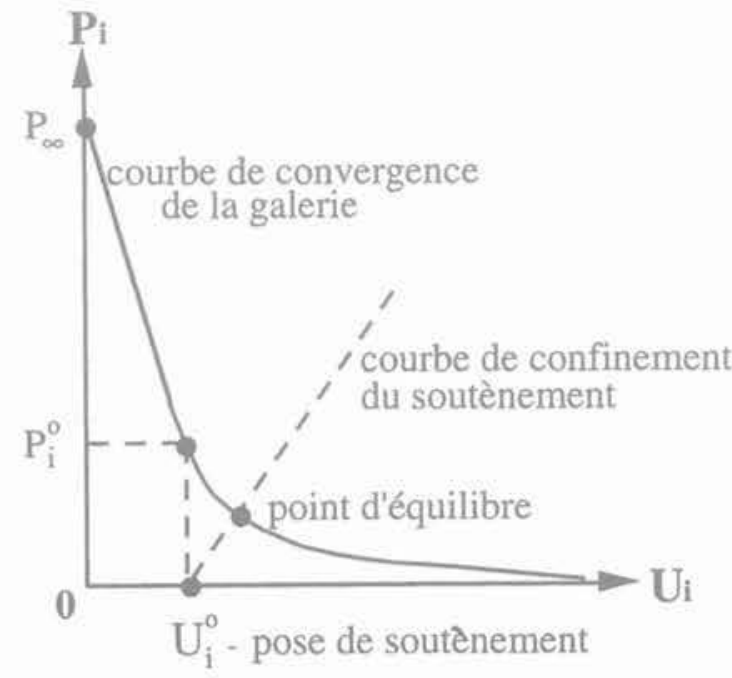

FiG. 22 Méthode de Convergence-Confinement classique.

Classical method of Convergence-Confinement. 
3. Déterminer la courbe de confinement de soutènement par voie théorique (AFTES, 1983) ou expérimentale (Bernaud, 1991). Dans le cas particulier d'un soutènement élastique, ceci revient à déterminer la raideur adimensionnelle $\mathrm{K}_{s^{\prime}}$, telle que :

$$
\left(\mathrm{P}_{\infty}^{*}-\Delta \mathrm{P}^{*}\right)=\mathrm{K}_{\mathrm{s}}^{*} \mathrm{E}^{*}\left(\mathrm{U}_{\mathrm{i}}-\mathrm{U}_{\mathrm{I}}^{\circ}\right)
$$

\section{Tracer la courbe de confinement partant du} point $\left(-P_{\infty}^{\prime}, E^{\prime \prime} U_{i}^{\alpha}\right)$, et relever les coordonnées du point d'intersection $\left(\Delta \mathrm{P}_{\mathrm{eq}}^{\prime}, \mathrm{E}^{\prime} \mathrm{U}_{1}^{\mathrm{eq}}\right)$ entre la courbe de confinement et la courbe de convergence du massif correspondant à la température estimée en paroi. Ces valeurs permettent de déduire la pression et la convergence à l'équilibre. Notons que pour un soutènement élastique, la courbe de confinement est une ligne

droite partant du point $\left(-\mathrm{P}_{\infty}^{*}, \mathrm{E}^{\prime} \mathrm{U}_{\mathrm{i}}^{0}\right)$ avec une pente égale à $\mathrm{K}_{\mathrm{s}}^{*}$ (voir Fig. 23). Une illustration graphique de cette procédure est donnée ci-dessous.

Comme on l'a déjà remarqué, le point d'équilibre ainsi déterminé n'est exact que si aucune décharge élastique n'a eu lieu pendant la phase transitoire, auquel cas on parcourt les mêmes phases comme celles décrites précédemment pour le trajet $\mathscr{L}$. Dans le cas contraire, un calcul exact doit prendre en compte les zones de décharge élastique, et le point d'équilibre ainsi déterminé n'est qu'approximatif.

A titre d'indication, prenons le cas d'une galerie de diamètre de $4 \mathrm{~m}$, située à $500 \mathrm{~m}$ de profondeur dans l'argile de l'Aisne, dont les caractéristiques sont celles du tableau II. On a donc :

$$
\begin{aligned}
& \mathrm{a}=2 \mathrm{~m} \\
& \mathrm{P}_{\infty}^{*}=\gamma \mathrm{HC}=1,7 \text { (en prenant } \gamma=20 \mathrm{kNm}^{-3}, \\
& \mathrm{E}^{*}=533 \\
& \mathrm{~T}^{*}=185^{\circ} \mathrm{C}
\end{aligned}
$$

Supposons que le soutènement soit assuré par une couche de béton projeté mise en place avant la phase d'échauffement, qui peut être assimilée à une coque élastique d'épaisseur $\mathrm{e}=0,2 \mathrm{~m}$. Dans ce cas, la rigidité du soutènement s'écrit de la manière suivante (AFTES, 1979, 1983) :

$$
K_{s}=\frac{E_{b} e}{\left(1-v_{b}^{2}\right) a}
$$

$E_{b}$ et $v_{b}$ le module d'Young et le coefficient de Poisson du béton projeté. On prendra $\mathrm{E}_{\mathrm{b}} \sim 15000 \mathrm{MPa}$ et $v_{\mathrm{b}} \sim 0,15$, soit donc :

$$
\mathrm{K}_{\mathrm{S}}=1535 \mathrm{MPa} \mathrm{c}^{\prime} \text { est-à-dire }: \mathrm{K}_{\mathrm{S}}^{*} \equiv \frac{\mathrm{K}_{\mathrm{S}}}{\mathrm{E}} \approx 0,5 \text {. }
$$

Supposons que la convergence acquise au moment de la pose du soutènement soit donnée par $\mathrm{U}_{1}^{\circ}=0,5 \%$, soit $\mathrm{E}^{*} \mathrm{U}^{\circ} \sim 2,7$, il est alors possible de tracer la courbe de confinement correspondante, comme l'illustre la figure 24.

En relevant les coordonnées des points d'intersection $\left(\Delta P_{e q}^{*}, E^{*} U_{i}^{e q}\right)$ entre la courbe de confine-

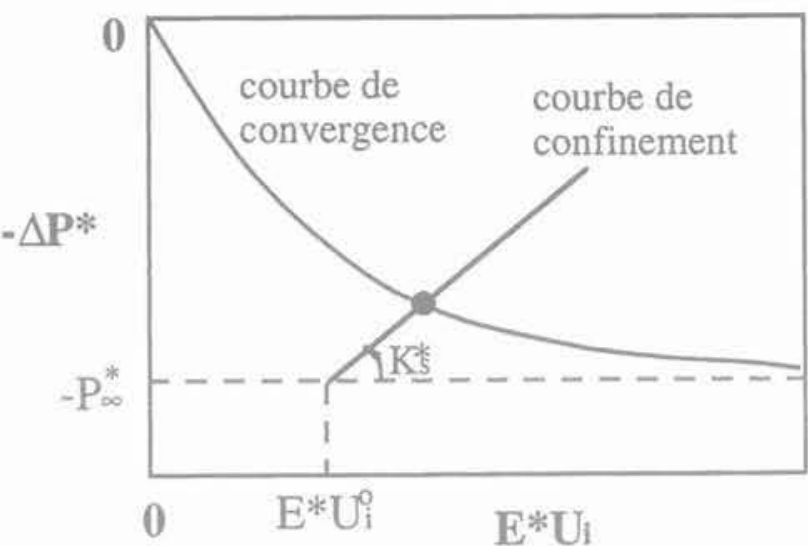

FIG.23 Convergence-Confinement avec chargement thermique.

Convergence-Confinement with thermal loading.

ment et les courbes de convergence, on peut estimer les valeurs et la pression exercée sur le soutènement $\mathrm{P}_{i}^{\text {eq }}$ d'après la formule $\mathrm{P}_{i}^{\mathrm{eq}}=\left(\mathrm{P}_{\infty}^{*}-\Delta \mathrm{P}_{\mathrm{eq}}^{*}\right) \mathrm{C}$, ainsi que la valeur de la convergence $\mathrm{U}_{i}^{e q}$ à l'état d'équilibre final et, en conséquence, le taux de contrainte $\sigma_{b}$ dans le béton projeté. Ces valeurs sont résumées dans le tableau ci-dessous.

Dans cet exemple, si l'influence du champ thermique sur la convergence peut paraître modérée (augmentation relative de $20 \%$ tout de même lorsque $\theta_{\text {}}$ passe de 0 à 0,8 ), l'accroissement de la pression quil s'exerce sur le soutènement, pour la mềme augmentation de température, est tout à fait importante. En effet, alors que le taux de contrainte de $5 \mathrm{MPa}$ (en l'absence de chargement thermique) sur le béton projeté reste acceptable, l'augmentation de température conduit rapidement au dépassement de la contrainte limite (inférieure à $10 \mathrm{MPa}$ ).

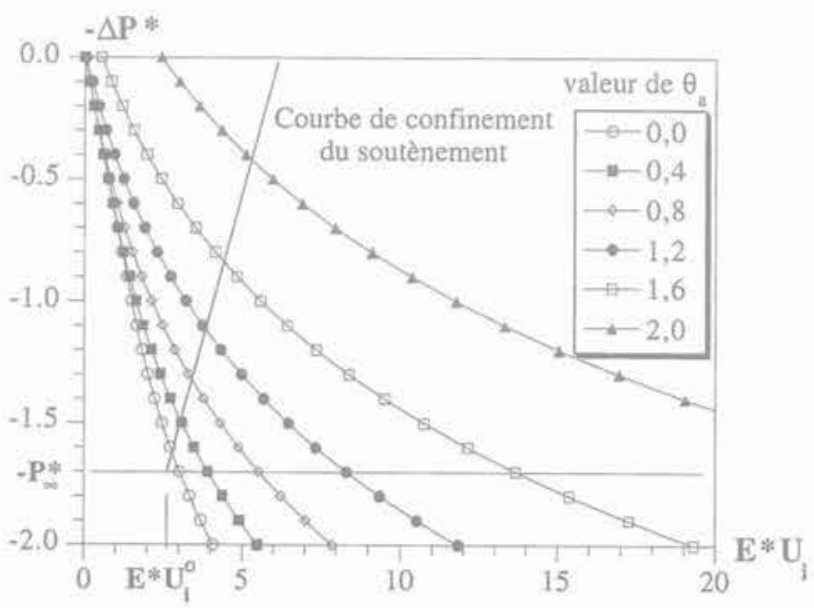

FIG. 24 Exemple d'application de l'abaque pour un prédimensionnement de soutènement des galeries soumises à un chargement thermique.

Example of application of the convergence graph of the preliminary dimensioning of the lining of a gallery under thermal loading. 


\begin{tabular}{c|c|c|c|c|c|c}
$\theta_{\mathrm{a}}$ & $\mathrm{T}_{\alpha}\left({ }^{\circ} \mathrm{C}\right)$ & $\mathrm{E}^{*} \mathrm{U}^{\mathrm{*q}}$ & $\mathrm{P}^{*}$ & $\mathrm{U}_{\mathrm{i}}^{\mathrm{*a}}(\%)$ & $\mathrm{P}^{\mathrm{*q}}(\mathrm{MPa})$ & $\sigma_{\mathrm{b}}(\mathrm{MPa})$ \\
\hline 0,0 & 0 & 2,8 & 1,62 & 0,53 & 0,5 & 5 \\
\hline 0,4 & 74 & 3,1 & 1,50 & 0,58 & 1,2 & 12 \\
\hline 0,8 & 148 & 3,4 & 1,33 & 0,64 & 2,2 & 22 \\
\hline 1,2 & 222 & 3,9 & 1,12 & 0,73 & 3,5 & 35 \\
\hline 1,6 & 296 & 4,4 & 0,85 & 0,83 & 5,1 & 51 \\
\hline 2,0 & 370 & 5,3 & 0,42 & 0,99 & 7,7 & 77 \\
\hline
\end{tabular}

En réalité, on peut penser que le soutènement doit atteindre la rupture rapidement, ce qui peut se traduire par une diminution progressive de la pente de la courbe de confinement (perte de rigidité), conduisant à davantage de convergence.

En pratique, on doit alors réexaminer le dimensionnement (augmenter l'épaisseur du béton projeté par exemple), ou considérer d'autres types de soutènement (béton projeté plus boulonnage ou cintres métalliques, etc.), de sorte que le taux de travail reste compatible avec la résistance du soutènement.

La simplicité de cette méthode permet dans ce cas d'examiner un grand nombre de choix techniques, en vue d'optimiser le prédimensionnement, dans de courts délais.

\section{5}

\section{Conclusions}

Pour appréhender le comportement puis le prédimensionnement d'une galerie souterraine soumise simultanément à une pression interne et à un échauffement en paroi, l'intérêt du modèle thermoélastoplastique original, présenté dans cet article et illustré par des applications pratiques, a été clairement mis en évidence.

On retiendra essentiellement les points suivants ; - Le modèle, quasiment explicite, est d'une grande simplicité d'emploi. De cette simplicité découle un grand nombre de déductions théoriques sur le comportement d'une galerie souterraine soumise à chargement thermique, et ce sans aucun recours à des calculs numériques.

D'autre part, grâce à cette simplicité, la solution analytique établie sert comme une solution de référence fiable.

- Pour les cas de chargement monotone, vérifiant (H1), (H2) et (H3), et lorsque l'échauffement est inférieur à un certain seuil (c'est le cas de tous les calculs menés dans cet article), l'état d'équilibre dépend uniquernent de la valeur finale des sollicitations et non pas de leurs histoires d'évolution.

- Sur le plan de l'exploitation numérique, l'état équilibre, à n'importe quel instant donné, peut être déterminé directement, sans avoir recours à un schéma d'intégration pas à pas dans le temps. Ceci permet entre autres de s'affranchir des problèmes délicats sur la longueur de pas de temps, et de la cumulation d'erreur. La performance qui en résulte rend l'exploitation numé- rique et les études paramétriques beaucoup plus efficaces.

Du point de vue du comportement thermomécanique d'une galerie circulaire, les études paramétriques ont montré que :

- Suivant l'élévation de la température, le chargement thermique peut engendrer des déplacements significatifs, voire plus importants que ceux dus aux sollicitations mécaniques. Pour l'étude des ouvrages souterrains soumis à des fortes variations de température, la prise en compte des effets thermiques est donc indispensable.

- Le chargement mécanique initial a une influence très significative sur le comportement de la galerie durant l'échauffement. Inversement, le chargement thermique modifie considérablement le champ de contraintes initial.

- Toute chose égale par ailleurs, la convergence thermique est une fonction croissante du module d'Young $E$, tandis que la convergence mécanique (sous condition isotherme) est une fonction décroissante de $\mathrm{E}$ ; la relation entre le module d'Young et la convergence totale dépend donc de l'intensité relative entre le chargement thermique et le chargement mécanique.

Cette constatation, propre à la thermomécanique, vient du fait que le module d'Young représente à la fois la rigidité du matériau et la constante de proportionnalité des contraintes thermiques.

Enfin, en s'appuyant sur les résultats du présent modèle, on peut l'étendre facilement aux cas suivants : - matériaux frottants obéissant au critère de MohrCoulomb (Wong, 1994);

- comportement du massif élasto-viscoplastique (Wong et Rousset, 1993).

\section{Annexe : Solution détaillée pour chaque phase}

\section{A.1. Phase 1}

$$
\begin{aligned}
& \sigma_{r}+P_{\infty}=\Delta P\left(\frac{a}{r}\right)^{2}-\frac{2}{r^{2}} \int_{a}^{r} \xi \theta(\xi, t) d \xi \\
& \sigma_{\theta}+P_{\infty}=-\left(\sigma_{r}+P_{\infty}\right)-2 \theta(r, t) \\
& \sigma_{2}+P_{\infty}=-2 \theta(r, t) \\
& E \frac{u}{r}=-(1+v)\left(\sigma_{r}+P_{\infty}\right)
\end{aligned}
$$




\section{A.2. Phase 2}

Zone élastique EL $(x<r<\infty)$

$$
\begin{aligned}
& \sigma_{r}+P_{\infty}=(1-\theta(x, t))\left(\frac{x}{r}\right)^{2}-\frac{2}{r^{2}} \int_{x}^{r} \xi \theta(\xi, t) d \xi \\
& \sigma_{\theta}+P_{\infty}=-\left(\sigma_{r}+P_{\infty}\right)-2 \theta(r, t) \\
& \sigma_{z}+P_{\infty}=-2 \theta(r, t) \\
& E \frac{u}{r}=-(1+v)\left(\sigma_{t}+P_{\infty}\right)
\end{aligned}
$$

Zone plastique RF $1(\mathrm{a}<\mathrm{r}<\mathrm{x})$

$$
\begin{aligned}
& \sigma_{r}+P_{\infty}=1-\theta(x, t)+\log \left(\frac{x}{r}\right)^{2}=\Delta P+\log \left(\frac{a}{r}\right)^{2}(A 9) \\
& \sigma_{\theta}=\sigma_{r}-2 \\
& \sigma_{2}+P_{\infty}=2 v\left[\log \left(\frac{x}{r}\right)^{2}-\theta(x, t)\right]-2(1-v) \theta(r, t)(A 11) \\
& E \frac{u}{r}=-2\left(1-v^{2}\right)\left[(1-\theta(x, t))\left(\frac{x}{r}\right)^{2}+\frac{2}{r^{2}} \int_{r}^{x} \xi \theta(\xi, t) d \xi\right. \\
& +(1+v)(1-2 v)\left[1-\theta(x, t)+\log \left(\frac{x}{r}\right)^{2}\right] \\
& \text { (A12) } \\
& E \lambda=2\left(1-v^{2}\right)\left[(1-\theta(x, t))\left(\frac{x}{r}\right)^{2}-(1-\theta(r, t))\right. \\
& \text { (A13) } \\
& +\frac{2}{r^{2}} \int_{r}^{x} \xi \theta(\xi, t) d \xi
\end{aligned}
$$

\section{A.3. Phase 3}

Zone élastique EL $(\mathrm{x}<\mathrm{r}<\infty)$ se référer aux expressions de la phase 2.

Zone plastique RF1 $(\mathrm{y}<\mathrm{r}<\mathrm{x}$ ) se référer aux expressions de la phase 2.

Zone plastique RA $(\mathrm{a}<\mathrm{r}<\mathrm{y})$

$$
\begin{array}{ll}
\sigma_{r}+P_{\infty}=1-\theta(x, t)+\log \left(\frac{x}{r}\right)^{2}=\Delta P+\log \left(\frac{a}{r}\right)^{2} & \text { (A14) } \\
\sigma_{\theta}=\sigma_{r}-2 & \text { (A15) } \\
\sigma_{2}=\sigma_{\theta} & \text { (A16) } \\
E \frac{u}{r}=-\frac{4\left(1-v^{2}\right)}{r^{2}} &
\end{array}
$$$$
\int_{y}^{x} \xi \theta(\xi, t) d \xi-\frac{6(1-v)}{r^{2}} \int_{y}^{x} \xi \theta(\xi, t) d \xi-2\left(1-v^{2}\right)
$$$$
-2\left(1-v^{2}\right)\left[(1-\theta(x, t))\left(\frac{x}{r}\right)^{2}-\left(1-\theta(x, t)\left(\frac{y}{r}\right)^{2}\right)\right]
$$

$$
\begin{aligned}
& -\frac{(1-2 v)}{2}\left[\begin{array}{l}
\left.(3 \Delta P-1)\left[\left(\frac{y}{r}\right)^{2}-1\right]-3\left(\frac{y}{r}\right)^{2}\right]^{\prime} \\
\log \left(\frac{y}{a}\right)^{2}+3 \log \left(\frac{r}{a}\right)^{2}
\end{array}\right] \\
& E \mu=(1-2 v) \log \left(\frac{y}{r}\right)^{2}+2(1-v)\left[\begin{array}{l}
\theta(r, t) \\
-\theta(y, t)
\end{array}\right] \\
& E \lambda=E \mu-E \frac{u}{r}
\end{aligned}
$$

A.4 Phase 4 ivalable uniquement pour $v=\frac{1}{2}$ )

Déplacement $(\mathrm{a}<\mathrm{r}<\infty)$

$$
E \frac{\mathrm{u}}{\mathrm{r}}=-3(1-\theta(x, \mathrm{t}))\left(\frac{\mathrm{x}}{\mathrm{r}}\right)^{2}-\frac{3}{\mathrm{r}^{2}} \int_{\mathrm{r}}^{\mathrm{x}} \xi \theta(\xi, \mathrm{t}) \mathrm{d} \xi
$$

Zone élastique EL $(x<r<\infty)$

$$
\begin{aligned}
& \sigma_{r}+P_{\infty}=2(1-\theta(x, t))\left(\frac{x}{r}\right)^{2}-\frac{2}{r^{2}} \int_{x}^{r} \xi \theta(\xi, t) d \xi \\
& \sigma_{\theta}+P_{\infty}=-\left(\sigma_{r}+P_{e}\right)-2 \theta(r, t) \\
& \sigma_{z}+P_{\infty}=-2 \theta(r, t)
\end{aligned}
$$

Zone plastique RF2 $(\mathrm{y}<\mathrm{r}<\mathrm{x})$

$$
\sigma_{r}+P_{\infty}=\frac{1}{2}\left[\begin{array}{l}
{[1-\theta(x, t)]\left[1+3\left(\frac{x}{r}\right)^{2}\right]} \\
\frac{3}{r^{2}} \int_{r}^{x} \xi \theta(\xi, t) d \xi-\int_{r}^{x} \frac{\theta(\xi, t)}{r} d \xi
\end{array}\right]
$$

$\sigma_{\theta}=\sigma_{r}-1-\theta(r, t)+E \frac{u}{r}$

$E \mu=\frac{3}{2}\left[\begin{array}{l}\sigma_{z}=\sigma_{r}-2 \\ {[1-\theta(x, t)]\left(\frac{x}{r}\right)^{2}-[1-\theta(r, t)]} \\ +\frac{1}{r^{2}} \int_{r}^{x} \xi \theta(\xi, t) d \xi\end{array}\right]$

\section{Zone plastique RA $(\mathrm{a}<\mathrm{r}<\mathrm{y})$}

$$
\begin{aligned}
& \sigma_{r}+\mathrm{P}_{\infty}=\Delta \mathrm{P}+\log \left(\frac{\mathrm{a}}{\mathrm{r}}\right)^{2} \\
& \sigma_{\theta}=\sigma_{\mathrm{r}}-2 \\
& \sigma_{2}=\sigma_{\theta} \\
& \mathrm{E} \mu=\theta(\mathrm{r}, \mathrm{t})-1 \\
& \mathrm{E} \lambda=E \mu-\mathrm{E} \frac{\mathrm{u}}{\mathrm{r}}
\end{aligned}
$$


Andra - Expression analytique simplifiée de la puissance thermique pour les déchets vitrifiés, rapport 770 NT 0502 . 1989.

AFTES - Stabilité des tunnels par la méthode convergence-confinement. Tunnels et Ouvrages souterrains, $n^{\circ} 32$, 1979,

AFTES - Recommandations sur l'emploi de la méthode convergence-confinement, Tunnels et Ouvrages souterrains, $n^{\circ} 59,1983$

Bérest P. - Point source of heat in an elastoplastic medium, J. Thermal Stresses, vol. $9, n^{\circ} 3,1986$.

Bérest P. et Rousset G. - Source of heat in an elastoviscoplastic medium, Thermomechanical couplings in solids, H.D. Bui and Q.S. Nguyen (Editors), 1987.

Bernaud D., Rousset G. - La nouvelle méthode implicite pour l'étude du dimensionnement des tunnels, Revue Française de Géotechnique, $n^{\circ}$ 60, pp. 5 26, 1992

Bernaud D., Rousset G. - L'essai du souténement à convergence contrôlée : résultats et modélisation, Proc. Int. Symp. Geotechnical Engineering of Hard Soils - Soft Rocks, Athènes, vol. 2, pp. 13811391, 1993.

Bland D.R. - Elastoplastic thick-walled tubes of work-hardening material subject to internal and external pressures and to temperature gradients, J. Mechanics and Physics of Solids, vol. 4, pp. 209229, 1956.

Booker J.R.. Savvidou C. - Consolidation around a point heat source, lnt. J. Numerical and Analytical Methods in Geomechanics, vol. 9, pp. 173-184, 1985.

Carslaw A.P., Jaeger C.J. - Conduction of heat in solids, Oxford Clarendon Press, 1960.

Corbetta F, Bernaud D., Nguyen Minh D., Contribution à la méthode Convergence-Confinement par le principe de la similitude, Revue Française de Géotechnique, $\mathrm{n}^{\circ} 54,1991$.
Djéran 1. La dilatation thermique des argiles, Gréco-Géomatériaux, rapport scientifique, pp. 559-566, 1992.

Egger P. - Influence du comportement post-rupture des roches sur le soutènement des tunnels, thèse $n^{\circ} 57$, Veroff. Inst. Bodenmech. Felsmech., Univ. Karlsruhe, traduction française BRGM $n^{\circ}$ 5401, 1973.

Giraud A. - Comportement thermo-hydromécaniques d'un puits de stockage de déchets radioactifs dans une argile profonde, Comptes rendus de Eurock'93, Lisboa, Portugal, vol. 1, pp. 293-300, 1993.

Godel J, - La Géothermie, Édition Doin. 1975.

Halphen B., Salençon J., Elasto-plasticité, Cours de calcul des structures anélastiques, Presses de l'ENPC, 1987.

Hildebrand F,B., Advanced calculus for applications, Prentice Hall, 1977.

Ishikawa H., Hata K., Thermoplastic creep stress analysis for a thick-walled tube, Int. J. Solids and Structure, vol. 16, pp. 291-299, 1980

Jain S.K. - Fundamental as-pects of the normality rule and their role in deriving constitutive laws of soils. Engineering Publications, P.O. Box 302 Blacksburg, Virginia 24060, 1980.

Jaeger J.C.- Numerical values for the temperature in radial heat flow, Int. J. Mathematics and Physics, vol. 34, 1956.

Mandel J. - Plasticité et poussée des terres. Cours de science des matériaux, fascicule II, ENSMP, 1976.

Mendelsen A.- Plasticity : theory and application, chapter 8, Macmillan series in applied mechanics, 1968.

Nguyen Minh D,- Analyse de la stabilité des galeries creusées dans une argile profonde par des modèles viscoplastiques, Journée sur les Argiles, ENSMP. 13 décembre, 1984.
Nguyen Minh D., Schmitt N.- Physical modelling for stability analysis of the linkage in deep underground gasification, 12th Symp. UCG, Sarrebrück, pp. 278-286, 1986 .

Nguyen Minh D., Schmitt N. - Stabilité mécanique du chenal de liaison dans la gazéficication souterraine du charbon. 6e Congrès international de Mécanique des Roches, Montrẻal, Canada, 1987.

Panet M. - Stabilité et soutenement des tunnels. La Mécanique des Roches appliquée aux ouvrages de gènie civil. Presses de l'ENPC, 1976.

Ritchie R.H. - Sakakura A.Y. - Asymptotique expansion of solutions of the heat equation in internally bounded cylindri cal geometry, J. Ap-plied Physics, vol 27, $\mathrm{n}^{\circ} 12,1956$.

Rousset G. - Comportement mécanique des argiles profondes. Application au stockage de dèchets radioactifs, thèse de doctorat de l'École Nationale des Ponts et Chaussées, 1988

Rousset G: - Lenfouissement de déchets radioactifs en formation géologique profonde, problèmes de Recherches Associées, ITBTP, n ${ }^{\circ}$ 507, pp. 101-113, 1992.

Wong H. - Un modèle analytique semiexplicite sur le comportement thermoélastoplastique des galeries souterraines dans les millieux argileux, Rapport G,3S $n^{\circ}$ 621-RP-G.3S-92-001, 1992

Wong H. Rousset G. - Thermoplastic behaviour of deep tunnels, Int. J. Thermal Stresses, Vol. 17. n³, 1994.

Wong H., Rousset G. - Comportement thermoplastique et thermo-viscoplas tique des galeries souterraines : solutions analytiques, 40 Colloque francopolonais de Geotechnique, Nancy, France, 1993.

Wong $\mathrm{H}$. - Comportement des galeries souterraines soumises à chargement thermique, thèse de doctorat de l'École Nationale des Ponts et Chaussées, 1994. 